\title{
Dynamic Stiffness Formulation for Free Vibration of Truncated Conical Shell and Its Combinations with Uniform Boundary Restraints
}

\author{
Chunyu Zhang, ${ }^{1}$ Guoyong Jin $\mathbb{D}^{\mathrm{D}}{ }^{2}$ Zhihao Wang, ${ }^{1}$ Xuqin Qian, ${ }^{1}$ and Linghua Tian ${ }^{2}$ \\ ${ }^{1}$ School of Energy and Power Engineering, Jiangsu University of Science and Technology, Zhenjiang 212003, China \\ ${ }^{2}$ College of Power and Energy Engineering, Harbin Engineering University, Harbin 150001, China \\ Correspondence should be addressed to Guoyong Jin; guoyongjin@hrbeu.edu.cn
}

Received 5 October 2020; Revised 9 December 2020; Accepted 19 December 2020; Published 7 January 2021

Academic Editor: Salvatore Caddemi

Copyright ( 12021 Chunyu Zhang et al. This is an open access article distributed under the Creative Commons Attribution License, which permits unrestricted use, distribution, and reproduction in any medium, provided the original work is properly cited.

\begin{abstract}
This paper presents a dynamic stiffness formulation for the free vibration analysis of truncated conical shell and its combinations with uniform boundary restraints. The displacement fields are expressed as power series, and the coefficients of the series are obtained as recursion formula by substituting the power series into the governing equations. Then, the general solutions can be replaced by an algebraic sum which contains eight base functions, which can diminish the number of degrees of freedom directly. The dynamic stiffness matrix is formulated based on the relationship between the force and displacement along the boundary lines. In the formulation, arbitrary elastic boundary restraints can be realized by introducing four sets of boundary springs along the displacement directions at the boundary lines. The modeling methodology can be easily extended to the combinations of conical shells with different thickness and semivertex angles. The convergence and accuracy of the present formulation are demonstrated by comparing with the finite element method using several numerical examples. Effects of the elastic boundary condition and geometric dimension on the free vibration characteristics are investigated, and several representative mode shapes are depicted for illustrative purposes.
\end{abstract}

\section{Introduction}

Rotatory shells are common components in practical engineering, which are widely used in construction, machinery, and ship industry. Dynamic behaviors of the shells have direct correlation with the stability, fatigue, and durability of the engineering structures. Therefore, a large amount of reports have been focused on the dynamic characteristic analysis of the rotatory shells [1-3]. Thereinto, most of the research is devoted to cylindrical shell rather than conical shell for the reason that the cylindrical shell is more widely used. Actually, conical shells are also significant components in many engineering applications, such as the shells of artificial satellites, submarines, and rockets. Thus, the dynamic modeling of the conical shell is indispensable. The coefficients of the governing equation are variable owing to the variation of radius along the generatrix direction, which results in the difficulty of deriving a closed-form solution for the vibration problem of conical shell. In recent years, the finite element method (FEM) has been well developed for vibration analysis of engineering structures. The vibration analysis of conical shell can be effectively conducted by the FEM [4-6], but, with regard to a more complex coupled structure (i.e., vessel and submarine), the FEM will encounter with inherent disadvantages on computational convergence and efficiency. Thus, developing an accurate and efficient modeling method can not only provide reliable analysis technique for vibration of an individual conical shell but also can afford theoretical basis for vibration modeling for coupled shell structures.

Different kinds of analytical and semianalytical methods have been proposed and developed for the vibration analysis of conical shell over the years. Irie et al. [7] proposed a transfer matrix method for free vibration of truncated 
conical shell. In this method, the governing equations are transformed into partial differential equation and the displacement and force are written as state vectors, and then the stiffness matrix can be obtained by solving the governing equation numerically. Based on the first-order of shear deformation theory, Hien et al. [8] developed the transfer matrix approach for vibration analysis of conical-conical shells. Wang et al. [9] proposed a precise transfer matrix method by combining the transfer matrix and the precise integration method for vibration and acoustics analysis of conical shell. The accuracy of transfer matrix method has been improved significantly by taking advantage of the precise integration method. Tong $[10,11]$ developed the vibration analysis model of conical shell by assuming the general solution in the form of power series. Similarly, by supposing the displacement shape function as power function, Kerboua et al. [12] derived exact general solutions of conical shell and obtained the mass and stiffness matrices using the framework of the FEM. Shu [13] carried out a dynamic analysis of conical shell based on a semianalytical method. In this method, the governing equation and boundary condition are discretized by generalized differential quadrature in the generatrix direction and Fourier expansion in the circumferential direction. Using the same method, Bagheri et al. [14] further conducted the vibration analysis of joined conical shell structure. Ni [15] provided a wave finite element method to interpret the type of waves that travel in the conical shell and discussed the vibration characteristics in terms of wave propagation. A discrete singular convolution method was reported by Civialek [16] for investigating the free vibration behaviors of a rotating conical shell with different kinds of material properties. Ansari et al. [17] proposed an efficient numerical method based on the Rayleigh-Ritz formulation to study the vibration problem of laminated composite conical shells.

In the analysis of vibration behaviors of combination of conical shells, as far as the authors know, only few published literatures have been reported. Stanley and Ganesan [18] proposed a semianalytical finite element method for free vibration analysis of conical shell and the combinations of conical shell with different semivertex angles (i.e., $0^{\circ}$ and $90^{\circ}$ ). Efraim and Eisenberger [19] studied the free vibration of conical shell and the segmented shells. Xie et al. [20] presented a unified analytic approach for dynamic analysis of stepped conical shell with discrete thickness and semivertex angles. Shakouri and Kouchakzadeh [21] performed analytical and experimental study to assess the free vibration characteristics of joined conical shells.

Most of the above reports are concerned with conical shell with specific boundary conditions or classical boundary conditions. In recent years, the artificial spring technique has been used by many researchers to model elastic boundary restrains and realize parameter modeling of boundary condition. Based on the energy variation method, Qu et al. [22] developed a domain decomposition method for vibration analysis of conical shell with elastic boundary condition by adopting the penalty function method. The modified Fourier series method was presented by Jin et al. [23] for dynamic analysis of conical shell with general boundary condition. Based on the Donnell thin shell theory, Song [24] developed a theoretical vibration model for the conical shell with elastic boundary restraints and studied the effect of added mass along the boundary lines.

In recent years, an elegant modeling method called the dynamic stiffness method (DSM) [25] has been well developed and widely used in vibration analysis of beams, plates and shells structures [26-36]. In the DSM, the frequency-dependent shape functions are derived directly from the governing equation. Thanks to the exactness of the shape functions, the DSM can offer results with good convergence and accuracy. However, not all kind of structures can obtain exact shape function from the governing equation. Thus, the DSM is mostly used in one-dimensional structure like beams $[26,27]$ and Levy-type plate [37]. Until the last few years, it is reported that the DSM has been used to establish the dynamic model of rectangular plate with general boundary conditions $[38,39]$. Research on dynamic stiffness formulation for shell structure is rarely reported, and only a few of papers are about cylindrical shell $[29,31]$. To the authors' best knowledge, the DSM has never been applied to vibration modeling for conical shell. This paper attempts to develop a dynamic stiffness formulation for vibration analysis of conical shell and its combinations with classical and elastic boundary conditions. In the formulation, the general solution is assumed as trigonometric functions in the circumferential coordinate and power series in the generatrix direction. The coefficients of the series are obtained in a recursion formula with the aid of governing equations. Based on the general solution, the expressions of the displacement and force along the boundary lines can be obtained. One can write the expressions in matrix form and extract the coefficients in vector form. By eliminating the coefficient vector, the dynamic stiffness matrix is formulated ultimately. Two kinds of boundary condition are considered: classical boundary condition and elastic restraint boundary condition. The elastic restraints are achieved by applying four groups of artificial springs at the end of the conical shell, and the boundary restraints can be adjusted by setting the stiffness values of the springs. Numerical examples are given to verify the convergency and correctness of the present formulation by comparing with the FEM. Meanwhile, the effects of geometric parameters and boundary restraints on the vibration characteristics are investigated. The main intention of the paper is to provide an elegant and efficient dynamic modeling approach for conical shell structures with uniform boundary conditions.

\section{Theoretical Formulation}

2.1. Governing Equation of Conical Shell. The truncated conical shell with uniform thickness and material is considered in this paper, and the sketch is shown in Figure 1. The coordinates in generatrix, circumferential, and normal direction are $x, \theta$, and $z$, respectively, and the displacements along the coordinates are donated as $u, v$, and $w$ in turn. $R_{0}$ and $L$ indicate the radius in the middle and length from end to the middle along the generatrix. The $x$ axis is along the generatrix whose origin is the middle of the shell. $\alpha$ is the 


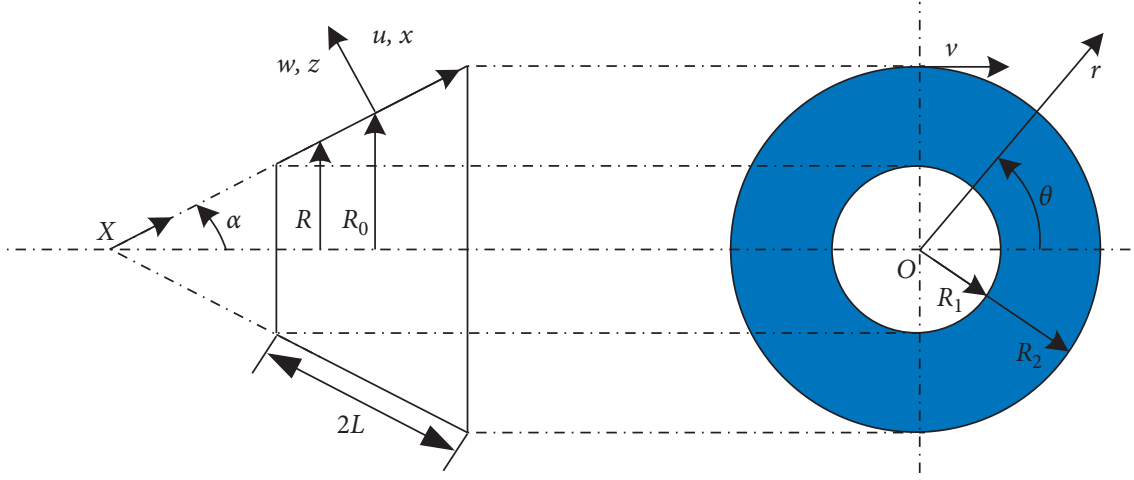

Figure 1: Sketch of geometry and coordinate system of the conical shell.

semivertex angle. Based on the Flügge thin shell theory, the governing equation of the conical shell can be expressed as

$$
\begin{array}{r}
\frac{\partial N_{x}}{\partial x}+\frac{1}{R(x)} \frac{\partial N_{x \theta}}{\partial \theta}+\frac{s}{R(x)}\left(N_{x}-N_{\theta}\right)-\rho h \frac{\partial^{2} u}{\partial t^{2}}=0, \\
\frac{\partial N_{x \theta}}{\partial x}+\frac{1}{R(x)} \frac{\partial N_{\theta}}{\partial \theta}+\frac{c}{R(x)} \frac{\partial M_{x \theta}}{\partial x}+\frac{c}{R^{2}(x)} \frac{\partial M_{\theta}}{\partial \theta}+2 \frac{s}{R(x)}-\rho h \frac{\partial^{2} v}{\partial t^{2}}=0, \\
\frac{\partial^{2} M_{x}}{\partial x^{2}}+\frac{2}{R(x)} \frac{\partial^{2} M_{x \theta}}{\partial x \partial \theta}+\frac{1}{R^{2}(x)} \frac{\partial^{2} M_{\theta}}{\partial \theta^{2}}+\frac{2 s}{R(x)} \frac{\partial M_{x}}{\partial x}-\frac{s}{R(x)} \frac{\partial M_{\theta}}{\partial x}-\frac{c}{R(x)} N_{\theta}-\rho h \frac{\partial^{2} w}{\partial t^{2}}=0,
\end{array}
$$

where $E$ and $v$ indicate elastic modulus and Poisson's ratio, $s=\sin \alpha, c=\cos \alpha$, and $R=R_{0}+x \sin \alpha$. The expressions of the stress resultants can be written as

$$
\begin{aligned}
N_{x}= & \frac{E h}{1-v^{2}}\left[\frac{\partial u}{\partial x}+v\left(\frac{1}{R} \frac{\partial v}{\partial \theta}+\frac{s}{R} u+\frac{c}{R} w\right)-k \frac{c}{R} \frac{\partial^{2} w}{\partial x^{2}}\right] \\
N_{x \theta}= & \frac{E h}{2(1+v)}\left[\frac{1}{R} \frac{\partial u}{\partial \theta}+\frac{\partial v}{\partial x}-\frac{s}{R} v\right. \\
& \left.+k\left(-\frac{c}{R^{2}} \frac{\partial^{2} w}{\partial x \partial \theta}+\frac{c^{2}}{R^{2}} \frac{\partial v}{\partial x}+\frac{c s}{R^{3}} \frac{\partial w}{\partial \theta}-\frac{c^{2} s}{R^{3}} v\right)\right] \\
N_{\theta}= & \frac{E h}{1-v^{2}}\left[v \frac{\partial u}{\partial x}+\frac{1}{R(x)} \frac{\partial v}{\partial x}+\frac{s}{R(x)} u+\frac{c}{R(x)} w\right] \\
M_{x}= & \frac{E h}{1-v^{2}}\left[-\frac{h^{2}}{12} \frac{\partial^{2} w}{\partial x^{2}}+\frac{h^{2}}{12} v\left(-\frac{1}{R^{2}(x)} \frac{\partial^{2} w}{\partial \theta^{2}}\right.\right. \\
& \left.\left.+\frac{c}{R^{2}(x)} \frac{\partial v}{\partial \theta}-\frac{s}{R(x)} \frac{\partial w}{\partial x}\right)\right]
\end{aligned}
$$

$$
M_{\theta}=\frac{E h^{3}}{12\left(1-v^{2}\right)}\left[-\frac{1}{R^{2}} \frac{\partial^{2} w}{\partial \theta^{2}}-\frac{s}{R} \frac{\partial w}{\partial x}-v \frac{\partial^{2} w}{\partial x^{2}}-\frac{c s}{R^{2}} u-\frac{c^{2}}{R^{2}} w\right]
$$

$$
\begin{aligned}
M_{x \theta} & =\frac{E h^{3}}{12(1+v)}\left(-\frac{1}{R} \frac{\partial^{2} w}{\partial x \partial \theta}+\frac{c}{R} \frac{\partial v}{\partial x}+\frac{s}{R^{2}} \frac{\partial w}{\partial \theta}-\frac{c s}{R^{2}} v\right) \\
V_{x} & =\frac{1}{R}\left(\frac{\partial\left(R M_{x}\right)}{\partial x}-M_{\theta} s+\frac{\partial M_{x \theta}}{\partial \theta}+\frac{\partial M_{x \theta}}{\partial \theta}\right) .
\end{aligned}
$$

Substituting the equations (4)-(9) into equations (1)-(3), one can obtain the partial differential equation of the conical shell as

$$
\begin{array}{r}
L_{11} u+L_{12} v+L_{13} w=0, \\
L_{21} u+L_{22} v+L_{23} w=0, \\
L_{31} u_{c}+L_{32} v_{c}+L_{33} w_{c}=0,
\end{array}
$$

where $L_{i j}$ are the partial differential operators, the detailed expressions of which are given in Appendix A. 
2.2. General Solution. Based on the separation of variables technique, the general solution can be assumed as

$$
\begin{aligned}
& u(x, \theta, t)=\sum_{m=0}^{\infty} U(x) \cos (m \theta) e^{i \omega t}, \\
& v(x, \theta, t)=\sum_{m=0}^{\infty} V(x) \sin (m \theta) e^{i \omega t}, \\
& w(x, \theta, t)=\sum_{m=0}^{\infty} W(x) \cos (m \theta) e^{i \omega t},
\end{aligned}
$$

where $m$ is the circumferential wave number, $\omega$ is the circular frequency, $u(x), v(x)$, and $w(x)$ are the displacement components along the axes in meridional, circumferential, and normal directions. As described in [10], the displacement components in $x$ direction can be written in power series as follows:

$$
\begin{gathered}
U(x)=\sum_{n=0}^{\infty} a_{n} x^{n}, \\
V(x)=\sum_{n=0}^{\infty} b_{n} x^{n}, \\
W(x)=\sum_{n=0}^{\infty} c_{n} x^{n},
\end{gathered}
$$

where, $a_{n}, b_{n}$, and $c_{n}$ are constants of the series. By substituting equation (13) into the governing equation and matching the coefficient of similar terms of $x^{n}$, one can obtain the recursion formula of the constants as

$$
\begin{aligned}
& a_{n+2}=\sum_{i=1}^{6} A_{1, i} a_{n-5+i}+\sum_{i=1}^{4} B_{1, i} b_{n-3+i}+\sum_{i=1}^{4} C_{1, i} a_{n-3+i}, \\
& b_{n+2}=\sum_{i=1}^{6} A_{2, i} a_{n-5+i}+\sum_{i=1}^{6} B_{2, i} b_{n-5+i}+\sum_{i=1}^{4} C_{2, i} a_{n-3+i}, \\
& c_{n+4}=\sum_{i=1}^{6} A_{3, i} a_{n-3+i}+\sum_{i=1}^{5} B_{3, i} b_{n-3+i}+\sum_{i=1}^{8} C_{3, i} a_{n-5+i},
\end{aligned}
$$

where $A_{l, i}, B_{l, i}$, and $C_{l, i}(l, i=1-8)$ are the coefficients of the recursion formula which can be found in [10]. By means of above recursion formula, all the constants $a_{n+2}, b_{n+2}$, and $c_{n+4}$ with $n>0$ can be derived from $a_{0}, a_{1}, b_{0}, b_{1}, c_{0}, c_{1}, c_{2}$, and $c_{3}$. Therefore, the power series in equation (13) can be rewritten as

$$
\begin{aligned}
U(x) & =\mathbf{u}_{m} \cdot \mathbf{x}_{m}, \\
V(x) & =\mathbf{v}_{m} \cdot \mathbf{x}_{m}, \\
W(x) & =\mathbf{w}_{m} \cdot \mathbf{x}_{m},
\end{aligned}
$$

where

$$
\begin{aligned}
& \mathbf{u}_{m}=\left[\begin{array}{lll}
u_{1, m}(x) & \ldots & u_{8, m}(x)
\end{array}\right], \\
& \mathbf{v}_{m}=\left[\begin{array}{llll}
v_{1, m}(x) & \ldots & v_{8, m}(x)
\end{array}\right] \text {, } \\
& \mathbf{w}_{m}=\left[\begin{array}{llll}
w_{1, m}(x) & \ldots & w_{8, m}(x)
\end{array}\right], \\
& \mathbf{x}_{m}=\left[\begin{array}{llllllll}
a_{0} & a_{1} & b_{0} & b_{1} & c_{0} & c_{1} & c_{2} & c_{3}
\end{array}\right]^{T},
\end{aligned}
$$

where $u_{i, m}(x), v_{i, m}(x)$, and $w_{i, m}(x)$ are equivalent shape function of the power series. In general, concrete expressions of the shape functions are difficult to derive for the reason that it will be quite lengthy as the serial number $n$ increases. But, the derivation can be performed in numerical software conveniently.

Based on the above derivation, the general solution can be given as

$$
\begin{aligned}
& \widehat{u}(x, \theta)=\sum_{m=0}^{\infty} \mathbf{u}_{m} \cdot \mathbf{x}_{m} \cos (m \theta), \\
& \widehat{v}(x, \theta)=\sum_{m=0}^{\infty} \mathbf{v}_{m} \cdot \mathbf{x}_{m} \sin (m \theta), \\
& \widehat{w}(x, \theta)=\sum_{m=0}^{\infty} \mathbf{w}_{m} \cdot \mathbf{x}_{m} \cos (m \theta) .
\end{aligned}
$$

2.3. Formulation of Dynamic Stiffness Matrix. Figure 2 shows the schematic diagram of displacement and force at boundary lines of the conical shell. The boundary lines $x=-L$ and $x=L$ are considered as two boundary nodes. For each circumferential number $m$, the displacement and force components at the boundary nodes $x=-L$ and $x=L$ can be written in vector form as

$$
\begin{aligned}
& \mathbf{q}_{m}=\left[\begin{array}{c}
U_{m}(-L, \theta) \\
V_{m}(-L, \theta) \\
W_{m}(-L, \theta) \\
\psi_{m}(-L, \theta) \\
U_{m}(L, \theta) \\
V_{m}(L, \theta) \\
W_{m}(L, \theta) \\
\psi_{m}(L, \theta)
\end{array}\right], \\
& \mathbf{Q}_{m}=\left[\begin{array}{c}
N_{x m}(-L, \theta) \\
N_{x \theta m}(-L, \theta) \\
V_{x m}(-L, \theta) \\
M_{x m}(-L, \theta) \\
N_{x m}(L, \theta) \\
N_{x \theta m}(L, \theta) \\
V_{x m}(L, \theta) \\
M_{x m}(L, \theta)
\end{array}\right],
\end{aligned}
$$

By introducing equation (15) and equations (4)-(10) and extracting the coefficients, above vectors can be rewritten as

$$
\begin{aligned}
\mathbf{q}_{m} & =\mathbf{D}_{m} \mathbf{C}_{m}, \\
\mathbf{Q}_{m} & =\mathbf{F}_{m} \mathbf{C}_{m},
\end{aligned}
$$

where 

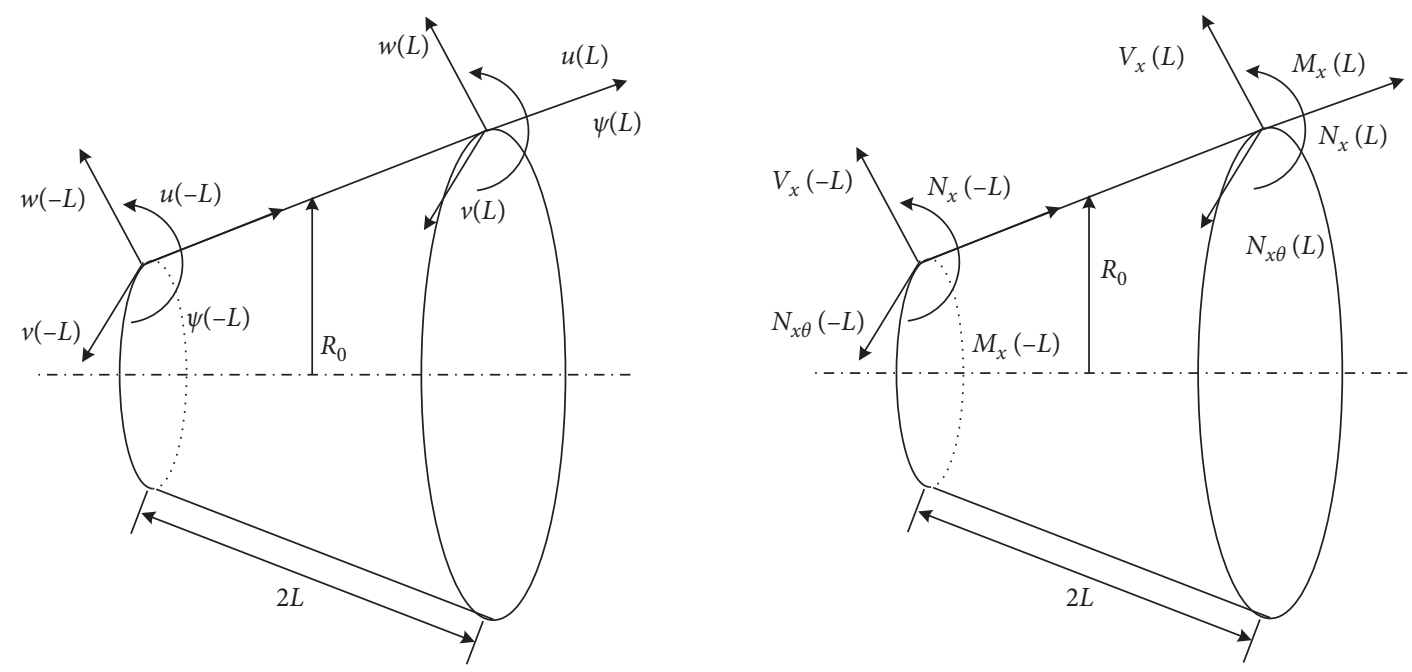

FIgURE 2: Displacement and force at boundary node of conical shell.

$$
\begin{aligned}
\mathbf{C}_{m} & =\left[\begin{array}{l}
a_{0} \\
a_{1} \\
b_{0} \\
b_{1} \\
c_{0} \\
c_{1} \\
c_{2} \\
c_{3}
\end{array}\right]_{8 \times 1} \begin{array}{lll} 
& \\
\mathbf{D}_{m} & =\left[\begin{array}{llll}
U_{m, 1}(-L) & \ldots & U_{m, 8}(-L) \\
V_{m, 1}(-L) & \ldots & V_{m, 8}(-L) \\
W_{m, 1}(-L) & \ldots & W_{m, 8}(-L) \\
\psi_{m, 1}(-L) & \ldots & \psi_{m, 8}(-L) \\
U_{m, 1}(L) & \ldots & U_{m, 8}(L) \\
V_{m, 1}(L) & \ldots & V_{m, 8}(L) \\
W_{m, 1}(L) & \ldots & W_{m, 8}(L) \\
\psi_{m, 1}(L) & \ldots & \psi_{m, 8}(L)
\end{array}\right]_{8 \times 8}, \\
\mathbf{F}_{m} & =\left[\begin{array}{cccc}
N_{x m, 1}(-L) & \ldots & N_{x m, 8}(-L) \\
N_{x \theta m, 1}(-L) & \ldots & N_{x \theta m, 8}(-L) \\
V_{x m, 1}(-L) & \ldots & V_{x m, 8}(-L) \\
M_{x m, 1}(-L) & \ldots & M_{x m, 8}(-L) \\
N_{x m, 1}(L) & \ldots & N_{x m, 8}(L) \\
N_{x \theta m, 1}(L) & \ldots & N_{x \theta m, 8}(L) \\
V_{x m, 1}(L) & \ldots & V_{x m, 8}(L) \\
M_{x m, 1}(L) & \ldots & M_{x m, 8}(L)
\end{array}\right]_{8 \times 8}
\end{array} .
\end{aligned}
$$

Eliminating the coefficient vector $\mathbf{C}_{m}$ from expressions in equation (19), the equation of motion and dynamic stiffness matrix of conical shell are formulated as

$$
\begin{aligned}
& \mathbf{Q}_{m}=\mathbf{K}_{m} \mathbf{q}_{m}, \\
& \mathbf{K}_{m}=\mathbf{F}_{m}\left(\mathbf{D}_{m}\right)^{-1} .
\end{aligned}
$$

The dynamic stiffness matrix $\mathbf{K}_{m}$ relates the displacement $\mathbf{q}_{m}$ and force $\mathbf{Q}_{m}$ vectors along the boundary nodes. The dimension of $\mathbf{K}_{m}$ matrix is $8 \times 8$.

2.4. Joined Conical Shell. In this section, the vibration modeling of joined conical shell is presented. Figure 3 shows the geometry and coordinate systems for the joined conical shell. For convenient of computation, the local coordinate systems of the conical shells can be transformed into a global coordinate system firstly. In the formulation, the coordinate of the left conical shell is set as the global coordinate system. The transformation of the displacement and forces from global to the global coordinate system can be expressed as

$$
\mathbf{X}_{g}=\mathbf{T X}
$$

where the subscript $g$ indicates global coordinate system and $\mathrm{T}$ is transformation matrix. According to the continuity condition of the displacement and force along the boundary lines shown in Figure 3(b), the relational expression can be obtained as

$$
\begin{aligned}
u_{1} & =-u_{2} \cos \left(\alpha_{1}-\alpha_{2}\right)+w_{2} \sin \left(\alpha_{1}-\alpha_{2}\right), \\
v_{1} & =v_{2}, \\
w_{1} & =u_{2} \sin \left(\alpha_{1}-\alpha_{2}\right)+w_{2} \cos \left(\alpha_{1}-\alpha_{2}\right), \\
\psi_{1} & =\psi_{2}, \\
N_{x, 1} & =-N_{x, 2} \cos \left(\alpha_{1}-\alpha_{2}\right)+V_{x, 2} \sin \left(\alpha_{1}-\alpha_{2}\right), \\
N_{x \theta, 1} & =N_{x \theta, 2}, \\
V_{x, 1} & =N_{x, 2} \sin \left(\alpha_{1}-\alpha_{2}\right)+V_{x, 2} \cos \left(\alpha_{1}-\alpha_{2}\right), \\
M_{x, 1} & =M_{x, 2} .
\end{aligned}
$$




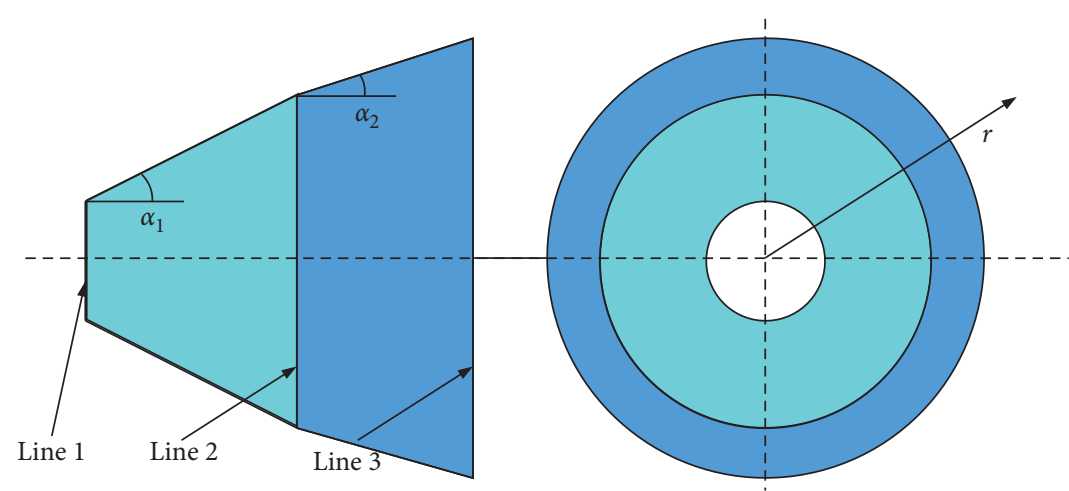

(a)

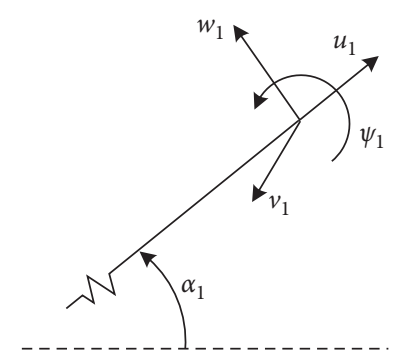

Conical shell 1

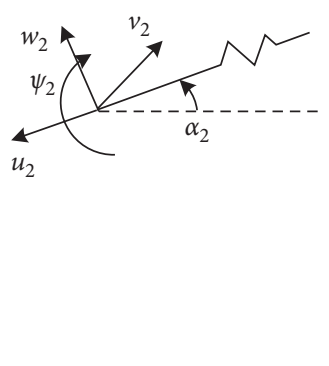

Conical shell 2

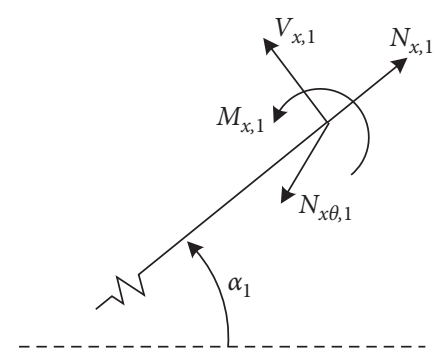

Conical shell 1

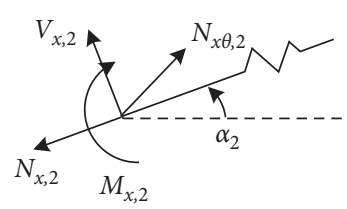

Conical shell 2

(b)

FIGURE 3: Sketches of (a) joined conical shell and (b) coordinating relationship of displacement and internal force along the coupling boundary of joined conical shell.

Therefore, the transformation matrix can be expressed as

$$
\mathbf{T}_{t}=\left[\begin{array}{cccccccc}
-\cos \delta \alpha & 0 & \sin \delta \alpha & 0 & & & & \\
0 & 1 & 0 & 0 & & 0 & & \\
\sin \delta \alpha & 0 & \cos \delta \alpha & 0 & & & & \\
0 & 0 & 0 & 1 & & & & \\
& & & & -\cos \delta \alpha & 0 & \sin \delta \alpha & 0 \\
& & & & 0 & 1 & 0 & 0 \\
& 0 & & & \sin \delta \alpha & 0 & \cos \delta \alpha & 0 \\
& & & & 0 & 0 & 0 & 1
\end{array}\right],
$$

$\delta \alpha=\alpha_{1}-\alpha_{2}$.

Using the transformation matrix, the dynamic stiffness matrix $\mathbf{K}_{g}$ in the global coordinate system can be obtained from the local coordinate system as

$$
\mathbf{K}_{g}=\mathbf{T}_{t} \mathbf{K T}_{t}^{T} .
$$

Before assembling two dynamic stiffness matrices of the conical shells, the boundary lines are numbered as shown in Figure 3. Then, the whole dynamic matrix for the jointed conical shell can be assembled by adopting the assembling procedure used in the FEM. Finally, the governing equation of the joined conical shell structure is expressed as

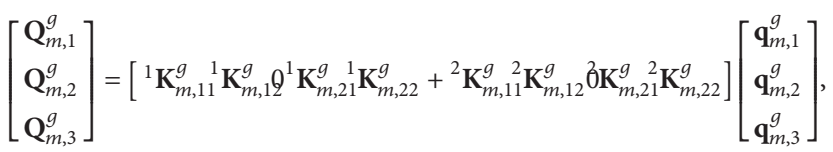

where the subscript $i(i=1,2,3)$ in the displacement and force vectors indicates boundary number and subscript $j$ $(j=1,2)$ in the stiffness matrices indicate the number of the substructure. In the above, only two substructures are taken for example to explain the assemble procedure of dynamic stiffness matrix. As to a more complex structure, the assemble procedure is the same. In view of cylindrical shell and annular plate are special cases for conical shell when $\alpha=0^{\circ}$ and $\alpha=90^{\circ}$, one can obtain the coupled structures like coupled conical shell-annular plate and coupled conicalcylindrical shell structure by setting one of $\alpha$ in Figure 3 as $90^{\circ}$ or $0^{\circ}$.

2.5. Application of Boundary Restraint. For the thin shell conical shell structure, both the classical boundary condition and elastic boundary condition are taken into account. Three types of classical boundary condition are assigned as follows:

Clamped edge (C): $u=0, v=0, w=0, \psi=0$

Simply supported edge (S): $N_{x}=0, v=0, w=0$, $M_{x}=0$

Free edge (F): $N_{x}=0, N_{x \theta}=0, V_{x}=0, M_{x}=0$ 
When applying boundary restraint, if there are zero elements in displacement vector $\mathbf{q}_{m}$, the corresponding row and columns of $\mathbf{K}_{m}$ will be removed. Application of the classical boundary condition is very concise which can further decrease the degree of system. But, it cannot be qualified to more complicated and variable boundary conditions which are often encountered in practical engineering problems. Thus, the elastic boundary restraints are further considered in this paper. Artificial spring technique is adopted to simulate the elastic boundary restraints along the boundary lines. Four sets of virtual springs used to restrain displacement components in the corresponding coordinate system. Arbitrary boundary restraints can be applied by adjusting the stiffnesses of the virtual springs. Therefore, the relations between boundary displacements and restraints can be defined as

$$
\begin{aligned}
N_{x} & =k_{u} u, \\
N_{x \theta} & =k_{v} v, \\
V_{x} & =k_{w} w, \\
M_{x} & =K_{\psi} \psi,
\end{aligned}
$$

where, $k_{u}, k_{v}, k_{w}$, and $K_{\psi}$ indicate the stiffness of the linear spring in $x, \theta$, and $r$ directions and rotational spring in $\psi$ direction, respectively.

The final equation of motion and dynamic stiffness matrix can be obtained after the application of boundary restraint. The natural frequency of the conical shell is calculated by

$$
\operatorname{det}\left|\mathbf{K}_{m}(\omega)\right|=0
$$

For convenient of numerical calculation, one can calculate the natural frequencies by searching the $\omega$ which makes the value of following expression infinite:

$$
\log \left(\frac{1}{\operatorname{det}\left|\mathbf{K}_{m}(\omega)\right|}\right)=0 .
$$

Once the natural frequency is determined, the corresponding mode shape can be computed by solving homogeneous equation in equation (21).

\section{Numerical Results}

\subsection{Free Vibration Analysis of Individual Conical Shell}

3.1.1. Convergence and Accuracy. In practical calculation, the power series in equation (13) should be numerically truncated so as to achieve the trade-off between convergence rate and computational expense. Therefore, the convergence study is performed firstly and to determine the reasonable truncated value for the power series. The geometric and material properties of the conical shell are used as follows: $R_{0}=1.5 \mathrm{~m}, \quad 2 L=2 \mathrm{~m}, \quad \alpha=\pi / 6, \quad h=0.01 \mathrm{~m}, \quad E=2.11 \mathrm{GPa}$, $\rho=7800 \mathrm{~kg} / \mathrm{m}^{3}$, and $v=0.3$.

Table 1 gives the first five orders of natural frequencies of conical shell with $\mathrm{C}-\mathrm{C}, \mathrm{C}-\mathrm{F}, \mathrm{S}-\mathrm{S}$, and $\mathrm{S}-\mathrm{C}$ boundary conditions, where the first letter indicates the boundary condition along boundary line $x=-L$ and the second letter indicates the boundary condition along boundary line $x=L$. The truncated number of the power series is assigned from 10 to 20 . From the results, it can be observed that the values of the frequencies decrease slightly as the truncated number increases. When the truncated number reaches to 14 , the values of frequencies are almost unchanged. Thus, it can be concluded than the fast convergence can be achieved by adopting a small number of power series in the formulation, and the computation cost for the present method is pretty low. Without special statement, the truncated number is fixed at 14 in the following examples.

To further demonstrate the accuracy and reliability of the present method, Table 2 compares the present results with results abstained from ANSYS software. In ANSYS software, SHELL 63 element is selected and the mesh number is $300 \times 100$. From Table 2, one can see that two sets of results agree well with each other. For directly comparing the results of the two methods, Figure 4 shows the relative errors of the first 40 frequencies. For the $\mathrm{C}-\mathrm{F}$ boundary condition, the errors are all less than $0.4 \%$. For the C-C boundary condition, all the errors are less than $0.5 \%$, except for the $34^{\text {th }}$ and $35^{\text {th }}$. The tiny errors can be attributed to the difference of the solving method. Several selected mode shapes of conical shell subject to $\mathrm{C}-\mathrm{F}$ and $\mathrm{C}-\mathrm{C}$ boundary conditions are depicted in Figures 5 and 6, which can illustrate the vibration feature of conical shell vividly and help us understand the vibration characteristics better. The number in the brackets $(n, m)$ represents axial and circumferential wave numbers, respectively.

Free vibration of conical shell with elastic boundary conditions is also conducted. It is assumed that the small radius end is clamped restrained and the large radius end is elastic restrained, while only one set of spring stiffness are fixed to $10^{3}$ or $10^{7}$ and the other three sets of spring stiffness are set to maximum. Table 3 gives natural frequencies of conical shell with elastic boundary conditions. Good agreement of two sets of results can be observed, which demonstrates the reliability of elastic boundary modeling in the present formulation. Almost of the relative errors are below $1 \%$, which can be attributed to the different calculation methods adopted. It can be concluded from the above numerical examples that the present method has the ability to conduct the vibration modeling and calculation of conical shell subject to uniform elastic boundary restraints in a unified manner.

Figure 7 depicts the variation curve of the first three frequencies of conical shell with different boundary conditions in response to circumferential number varying from 1 to 15 . The curves have a nearly uniform trend in those figures, where the frequencies firstly decrease, after reaching their minimum, then increase monotonously. It is noted that, as the axial wave number increases, the inflection points of the curves move right obviously. In addition, the maximum difference of the curves appears near the inflection points. When the conical shell is subject to $\mathrm{C}-\mathrm{F}$ boundary condition, the initial value of the frequency corresponding to $n=1$ is very small, and the increase and decrease tendencies of the curves are relatively flat. But, as the boundary restraint is enforced, the change rate of the frequency value for $n=1$ 
TABLE 1: Frequencies of the conical shell under different boundary conditions.

\begin{tabular}{|c|c|c|c|c|c|c|c|}
\hline \multirow{2}{*}{ Boundary condition } & \multirow{2}{*}{ Mode number } & \multicolumn{6}{|c|}{$f(\mathrm{~Hz})$} \\
\hline & & $N=10$ & $N=12$ & $N=14$ & $N=16$ & $N=18$ & $N=20$ \\
\hline \multirow{5}{*}{$\mathrm{C}-\mathrm{C}$} & 1 & 102.22 & 102.14 & 102.11 & 102.11 & 102.11 & 102.11 \\
\hline & 2 & 104.33 & 104.32 & 104.32 & 104.32 & 104.32 & 104.32 \\
\hline & 3 & 109.01 & 108.85 & 108.84 & 108.84 & 108.84 & 108.84 \\
\hline & 4 & 112.81 & 112.80 & 112.79 & 112.78 & 112.78 & 112.78 \\
\hline & 5 & 125.52 & 125.46 & 125.45 & 125.45 & 125.45 & 125.45 \\
\hline \multirow{5}{*}{$\mathrm{C}-\mathrm{F}$} & 1 & 28.54 & 28.54 & 28.52 & 28.51 & 28.51 & 28.51 \\
\hline & 2 & 32.12 & 32.12 & 32.11 & 32.10 & 32.10 & 32.09 \\
\hline & 3 & 32.22 & 32.19 & 32.18 & 32.18 & 32.18 & 32.18 \\
\hline & 4 & 39.94 & 39.92 & 39.90 & 39.90 & 39.90 & 39.90 \\
\hline & 5 & 46.14 & 46.09 & 46.08 & 46.05 & 46.06 & 46.06 \\
\hline \multirow{5}{*}{ S-S } & 1 & 78.62 & 78.61 & 78.59 & 78.59 & 78.59 & 78.59 \\
\hline & 2 & 78.75 & 78.79 & 78.81 & 78.80 & 78.80 & 78.80 \\
\hline & 3 & 87.10 & 87.06 & 87.03 & 87.03 & 87.03 & 87.03 \\
\hline & 4 & 91.64 & 91.62 & 91.62 & 91.61 & 91.61 & 91.61 \\
\hline & 5 & 99.32 & 99.30 & 99.29 & 99.28 & 99.28 & 99.28 \\
\hline \multirow{5}{*}{$\mathrm{S}-\mathrm{C}$} & 1 & 95.64 & 95.61 & 95.61 & 95.60 & 95.60 & 95.59 \\
\hline & 2 & 99.51 & 99.49 & 99.49 & 99.48 & 99.48 & 99.48 \\
\hline & 3 & 100.83 & 100.82 & 100.82 & 100.81 & 100.81 & 100.81 \\
\hline & 4 & 111.59 & 111.56 & 111.56 & 111.54 & 111.54 & 111.54 \\
\hline & 5 & 115.57 & 115.55 & 115.55 & 115.53 & 115.53 & 115.53 \\
\hline
\end{tabular}

TABLe 2: Frequencies of the conical shell under C-F and C-C boundary conditions ( $\mathrm{Hz})$.

\begin{tabular}{|c|c|c|c|c|c|c|c|c|c|c|c|}
\hline \multirow{2}{*}{$m$} & \multirow{2}{*}{ Method } & \multicolumn{5}{|c|}{$\mathrm{C}-\mathrm{F}$} & \multicolumn{5}{|c|}{$\mathrm{C}-\mathrm{C}$} \\
\hline & & 1 & 2 & 3 & 4 & 5 & 1 & 2 & 3 & 4 & 5 \\
\hline \multirow{2}{*}{1} & DSM & 140.8 & 371.8 & 408.7 & 447.9 & 491.0 & 377.2 & 413.6 & 448.4 & 492.5 & 530.4 \\
\hline & FEM & 141.0 & 371.3 & 408.1 & 449.2 & 489.1 & 377.1 & 414.6 & 452.7 & 493.0 & 531.7 \\
\hline \multirow{2}{*}{2} & DSM & 76.2 & 312.3 & 382.5 & 422.9 & 466.7 & 275.8 & 384.7 & 423.9 & 467.6 & 510.7 \\
\hline & FEM & 76.4 & 312.1 & 381.8 & 423.8 & 467.1 & 275.9 & 384.6 & 426.7 & 469.8 & 511.6 \\
\hline \multirow{2}{*}{3} & DSM & 46.0 & 228.6 & 353.2 & 398.7 & 443.1 & 203.5 & 334.4 & 396.7 & 443.9 & 491.3 \\
\hline & FEM & 46.2 & 228.4 & 353.3 & 398.5 & 443.5 & 203.8 & 334.1 & 397.2 & 445.1 & 493.0 \\
\hline \multirow{2}{*}{4} & DSM & 32.1 & 169.7 & 311.4 & 372.9 & 420.3 & 156.4 & 281.5 & 364.2 & 418.4 & 470.2 \\
\hline & FEM & 32.2 & 169.6 & 311.2 & 372.5 & 420.6 & 156.8 & 281.0 & 364.0 & 420.3 & 472.9 \\
\hline \multirow{2}{*}{5} & DSM & 28.5 & 132.8 & 268.5 & 345.9 & 400.2 & 126.3 & 237.9 & 330.5 & 395.5 & 451.6 \\
\hline & FEM & 28.4 & 132.6 & 268.1 & 345.4 & 399.5 & 126.7 & 238.4 & 329.8 & 395.7 & 454.2 \\
\hline \multirow{2}{*}{6} & DSM & 32.1 & 111.9 & 233.2 & 319.3 & 382.7 & 108.8 & 207.1 & 300.3 & 373.4 & 436.2 \\
\hline & FEM & 32.0 & 111.8 & 233.7 & 318.6 & 381.8 & 109.2 & 206.6 & 299.4 & 372.7 & 437.4 \\
\hline \multirow{2}{*}{7} & DSM & 39.9 & 104.0 & 208.6 & 296.5 & 366.3 & 102.1 & 187.0 & 276.7 & 354.4 & 424.0 \\
\hline & FEM & 39.9 & 103.9 & 208.1 & 295.7 & 365.3 & 102.4 & 186.6 & 275.6 & 353.2 & 423.5 \\
\hline \multirow{2}{*}{8} & DSM & 50.0 & 106.3 & 195.0 & 280.2 & 353.8 & 104.2 & 177.3 & 261.1 & 340.3 & 415.3 \\
\hline & FEM & 49.9 & 106.1 & 194.5 & 279.2 & 352.8 & 104.5 & 176.9 & 259.9 & 338.8 & 413.3 \\
\hline \multirow{2}{*}{9} & DSM & 61.5 & 115.8 & 192.0 & 271.6 & 346.5 & 112.8 & 177.1 & 254.0 & 332.4 & 411.0 \\
\hline & FEM & 61.5 & 115.6 & 191.5 & 270.6 & 345.5 & 113.0 & 176.8 & 252.9 & 330.8 & 407.8 \\
\hline \multirow{2}{*}{10} & DSM & 74.5 & 129.7 & 198.2 & 271.4 & 345.3 & 125.4 & 185.1 & 255.5 & 331.4 & 411.6 \\
\hline & FEM & 74.4 & 129.8 & 197.8 & 270.4 & 344.4 & 125.6 & 184.7 & 254.4 & 329.8 & 407.8 \\
\hline
\end{tabular}

obviously speeds up. The curves in Figures $7(\mathrm{c})$ and $7(\mathrm{~d})$ become almost identical when the boundary condition subject to $\mathrm{S}-\mathrm{C}$ and $\mathrm{C}-\mathrm{C}$.

3.1.2. Parameter Studies. In what follows, parameter studies are conducted to investigate the effects of geometric parameters on vibration characteristics of the conical shell structure. The half-cone angle is critical parameter of conical shell structure; thus, the effects of half-cone angle on frequency are first considered. The following geometric parameters are considered: $R_{1}=2 \mathrm{~m}, L=\sqrt{3} \mathrm{~m}, h / R_{0}=0.005$, and $\alpha=10^{\circ}-80^{\circ}$. Table 4 presents the natural frequencies of conical shell with different half-cone angles under $\mathrm{C}-\mathrm{F}, \mathrm{S}-\mathrm{S}$, $\mathrm{S}-\mathrm{C}$, and $\mathrm{C}-\mathrm{C}$ boundary conditions. To make it more intuitive, the data in Table 4 are further depicted as curves given in Figure 8. It is observed that frequencies are monotone decreasing for the case of C-F boundary condition. When the conical shell is subject to S-S boundary condition, the first frequency increases rapidly from a small value and reaches the maximum when $\alpha=50^{\circ}$. The rest orders of frequencies nearly remain unchanged as $\alpha$ 


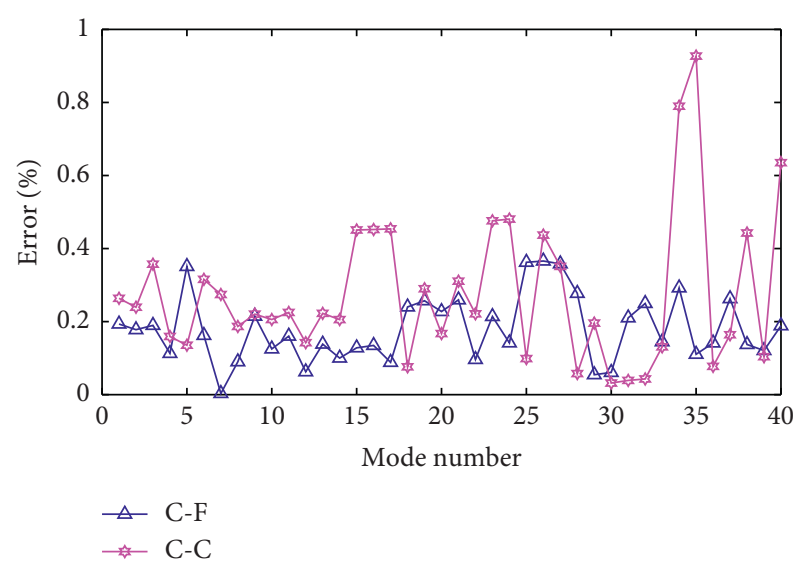

FIgURE 4: Relative deviation of the frequency of conical shell under $\mathrm{C}-\mathrm{F}$ and $\mathrm{C}-\mathrm{C}$ boundary conditions.

increases from $10^{\circ}$ to $50^{\circ}$, while decrease obviously as $\alpha$ exceeds $50^{\circ}$. As to $\mathrm{S}-\mathrm{C}$ and $\mathrm{C}-\mathrm{C}$ boundary conditions, the curves in Figures 8(c) and 8(d) have a similar tendency. When the orders of the frequencies are small, the frequencies increase slightly as $\alpha$ varies from $10^{\circ}$ to $50^{\circ}$, while the frequencies decrease slightly as the order reaches 5 or 6 . There are apparent decline in frequencies when the value of $\alpha$ exceeds $50^{\circ}$. On the whole, the value of frequency reaches minimum as $\alpha=80^{\circ}$. The main reason is that the conical shell is close to an annular plate when the half-cone angle is very large, and the coupling strength of in-plane and transverse motions of the conical shell is reduced dramatically, which directly results in the degradation of structural stiffness.

New examples are conducted to study the effect of length variation on the frequency of conical shell. Firstly, we define the length-diameter ratio as $L \sin \alpha / R_{1}$, and the ratio varies from 0.1 to 0.8 in the calculations. Comparison of frequencies of conical shell with different length-diameter ratios and different boundary conditions is presented in Table 5. In can be observed from the data, the large the length-diameter ratio is, the smaller the frequencies are. Only in rare cases, frequencies increase as the length-diameter ratio increases from 0.7 to 0.8 . For further investigation, the variation of the first three orders of frequencies in relation to length-diameter ratio is drawn in Figure 9. It can be observed that the trend of the curves for $\mathrm{C}-\mathrm{F}, \mathrm{C}-\mathrm{C}$, and $\mathrm{S}-\mathrm{C}$ boundary conditions are very similar; in addition, the values of the first three frequencies are very close. Only for S-S boundary condition, the differences among the frequencies are distinct, especially when the length-diameter ratio is small. On the whole, the frequencies decrease rapidly as the length-diameter ratio is small. As the length-diameter ratio increases gradually, the degradation rate of frequency becomes slower. That is to say, as the lengthdiameter ratio increases, its effect on the frequencies gradually diminishes.

Then, the influence of elastic boundary restraint on the natural frequency of the conical shell is studied. For convenience, we firstly define the nondimensional spring stiffness as $\bar{k}=\log (k / E h /(1-v))$ and rewrite the nondimensional spring stiffness in the four displacement directions as $\bar{k}_{u}, \bar{k}_{v}, \bar{k}_{w}$, and $\bar{K}_{\psi}$. In the numerical examples, boundary along $x=-L$ of the conical shell is clamped restrained, and two types of elastic boundary restraints are considered along $x=L$ : (1) one set of boundary spring stiffness is assumed to increase constantly from 0 to infinite and the other three sets are fixed to 0 ; (2) one set of boundary spring stiffness is assumed to increase constantly from 0 to infinite and the other three sets are fixed to infinite. Frequencies of conical shell subject to these two types of elastic boundary conditions are shown in Figures 10 and 11, respectively. In Figure 10, the origins of the curves in the four subfigures are coincident, because the boundary condition can be regarded as $\mathrm{C}-\mathrm{F}$ when the stiffnesses of the springs are infinitesimal. That can be verified by comparing frequency values of the origins with those in Table 2. On the whole, the curves can be divided into three parts: horizontal part, ascending part, and horizontal part. In the first horizontal part, the boundary is almost unrestrained because the stiffnesses are too minute. Then, in the ascending part, the elastic restraint starts to work and the frequencies increase rapidly. When the stiffness of the spring is large enough, the displacement can be viewed as fully clamped, so the curves turn to straight lines. The ascending part can be regarded as elastic restrained range. It is noted that the stiffness ranges corresponding to those elastic restrained range in the four subfigures are slightly different, which are $[-2,-1],[-3,1],[-3,1]$, and $[-5$, $-1]$. In addition, the increasing magnitudes of frequencies in the elastic restrained range are different too. When the displacement in $v$ direction is clamped, the frequencies are maximal. While the frequencies are almost unaffected when the displacement in $\psi$ direction is clamped. That is to say, the sensitivity of frequency to elastic restraint in different displacement directions is not the same. Next, we discuss the second set of elastic boundary condition. From Figure 11, the tendency of the curves is similar to those in Figure 10. At the beginning, three sets of displacements have been clamped, so the frequency values at the origins are relatively high. As the rest displacement is restrained, the increasing magnitudes of frequencies are relatively smaller than those in last the case. In the horizontal part, the boundary condition turns to $\mathrm{C}-\mathrm{C}$ since the stiffness of boundary spring is large enough. With the increase of stiffness, frequencies in Figures 11(a) and 11(b) increase obviously, whereas frequencies in Figures 11(c) and 11(d) nearly unchanged. In summary, frequencies are more sensitive to restraints in $u, v$, and $w$ directions than in $\psi$ direction.

3.2. Free Vibration Analysis of Jointed Conical Shell. In this section, the vibration analysis of jointed conical shell is performed. Firstly, vibration characteristics of the stepped jointed conical shell made of different thickness are studied. The geometric parameters of the stepped jointed conical shell used are $R_{0}=1.25 \mathrm{~m}, L=3 \mathrm{~m}$, and $\alpha=\pi / 6$. The conical shell is divided into three segments evenly along the axial direction and the thickness is defined as $h_{1}, h_{2}$, and $h_{3}$. Three sets of models are taken into consideration: (1) model 1 , $h_{1}=0.005 \mathrm{~m}, h_{2}=0.01 \mathrm{~m}$, and $h_{3}=0.015 \mathrm{~m}$; (2) model 2, $h_{1}=0.015 \mathrm{~m}, h_{2}=0.01 \mathrm{~m}$, and $h_{3}=0.005 \mathrm{~m}$; (3) model 3, $h_{1}=0.01 \mathrm{~m}, h_{2}=0.01 \mathrm{~m}$, and $h_{3}=0.01 \mathrm{~m}$. 


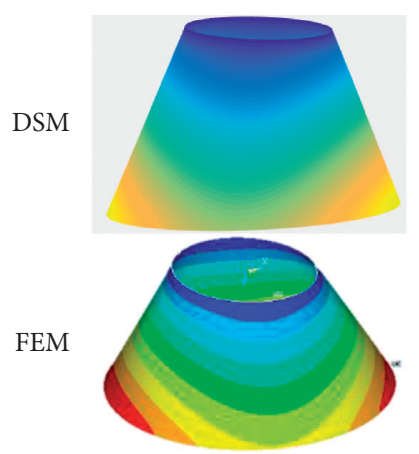

$(1,1)$
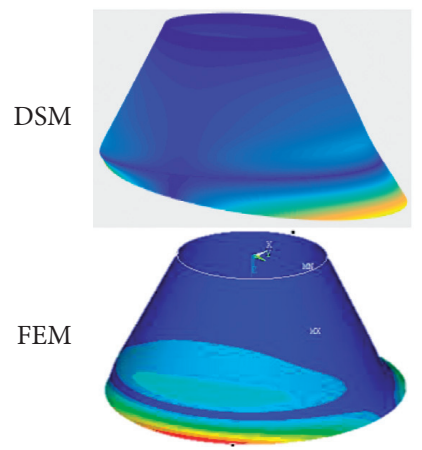

$(2,1)$

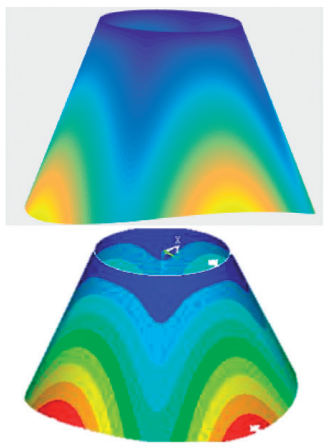

$(1,2)$
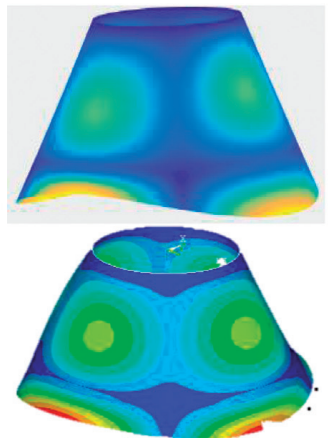

$(2,2)$

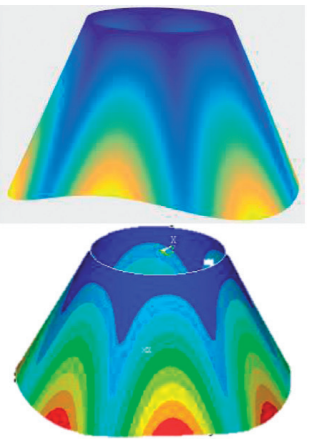

$(1,3)$
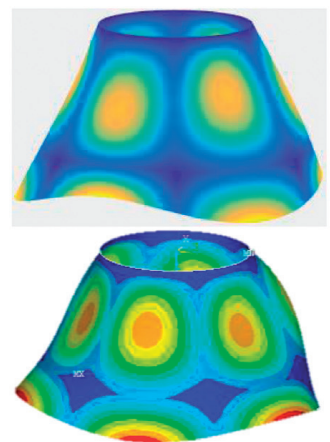

$(2,3)$

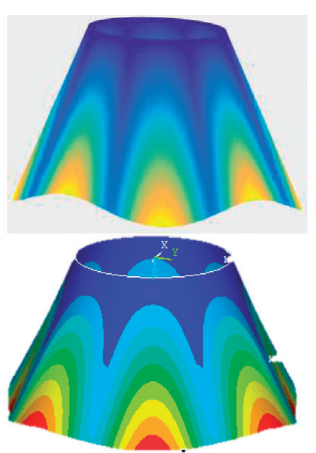

$(1,4)$

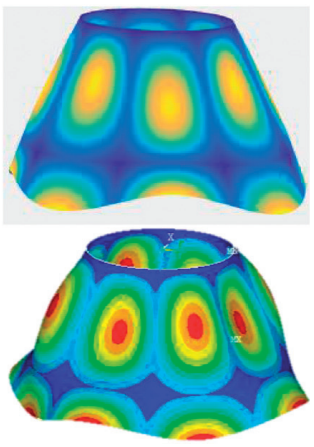

$(2,4)$

FIgUre 5: Mode shapes of conical shell under C-F boundary condition.
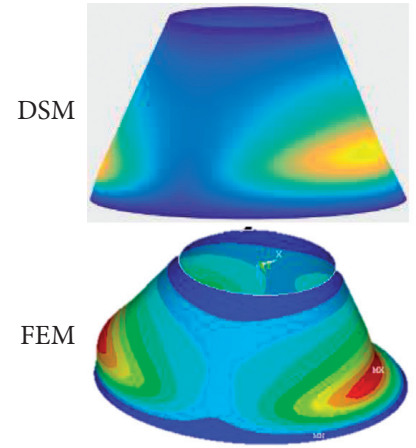

$(1,1)$

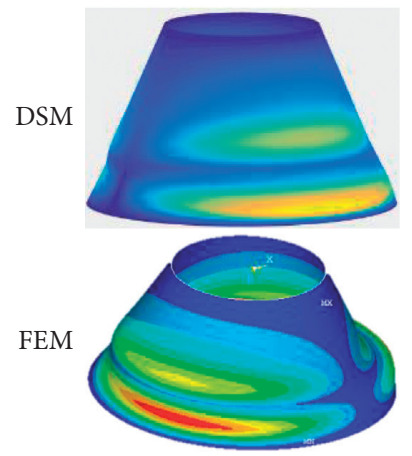

$(2,1)$

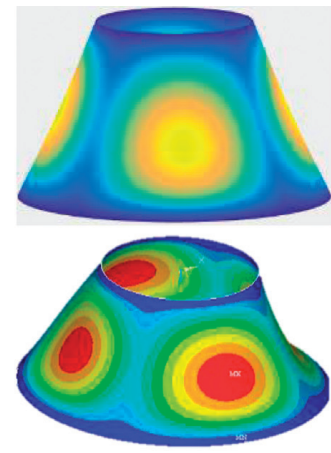

$(1,2)$

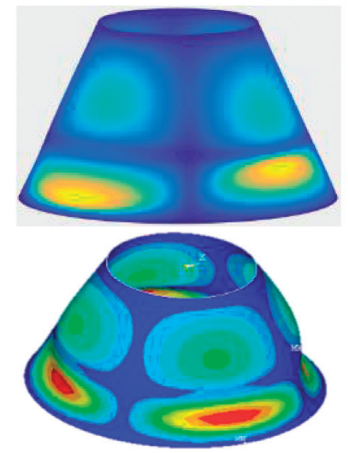

$(2,2)$

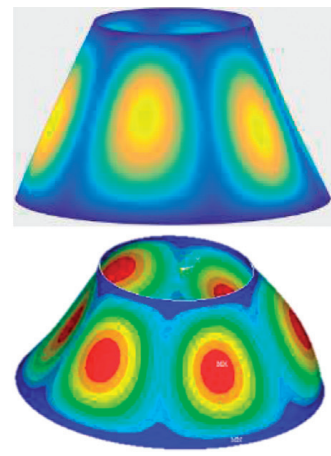

$(1,3)$
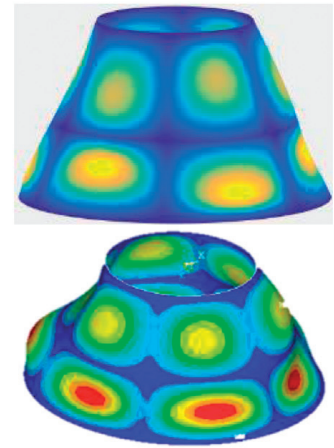

$(2,3)$

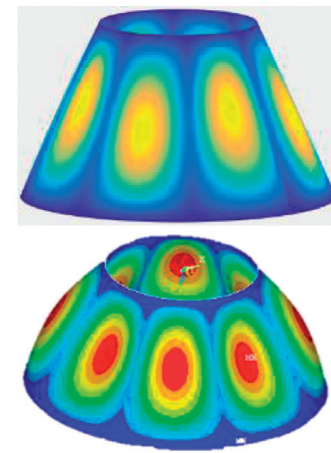

$(1,4)$

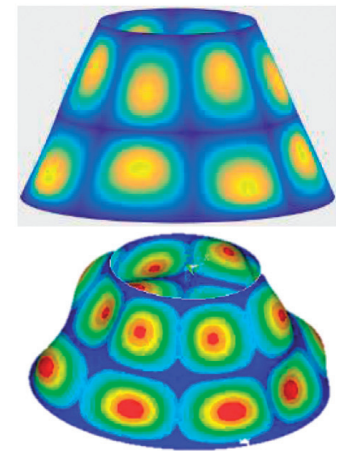

$(2,4)$

Figure 6: Mode shapes of conical shell under C-C boundary condition. 
TABLE 3: Frequency comparison of conical shell under elastic boundary conditions (Hz).

\begin{tabular}{|c|c|c|c|c|c|c|c|c|c|}
\hline $\begin{array}{l}\text { Spring } \\
\text { stiffness }\end{array}$ & Mode number & DSM & FEM & Error (\%) & $\begin{array}{c}\text { Spring } \\
\text { stiffness }\end{array}$ & Mode number & DSM & FEM & Error (\%) \\
\hline \multirow{6}{*}{$k_{u}$} & 1 & 17.69 & 17.71 & 0.11 & \multirow{6}{*}{$k_{w}$} & 1 & 67.13 & 67.13 & 0.00 \\
\hline & 2 & 19.98 & 19.88 & 0.50 & & 2 & 73.46 & 73.46 & 0.00 \\
\hline & 3 & 28.61 & 28.61 & 0.00 & & 3 & 77.53 & 77.45 & 0.10 \\
\hline & 1 & 19.68 & 19.68 & 0.00 & & 1 & 67.21 & 67.16 & 0.07 \\
\hline & 2 & 27.32 & 26.79 & 1.94 & & 2 & 73.53 & 73.43 & 0.14 \\
\hline & 3 & 29.02 & 29.44 & 1.45 & & 3 & 77.63 & 77.39 & 0.31 \\
\hline \multirow{6}{*}{$10^{3}$} & 1 & 66.60 & 66.41 & 0.29 & \multirow{4}{*}{$10^{3}$} & 1 & 67.21 & 67.26 & 0.07 \\
\hline & 2 & 73.18 & 73.13 & 0.07 & & 2 & 73.49 & 73.52 & 0.04 \\
\hline & 3 & 74.72 & 73.95 & 1.03 & & 3 & 77.67 & 77.70 & 0.04 \\
\hline & 1 & 58.65 & 58.46 & 0.32 & & 1 & 67.53 & 67.30 & 0.34 \\
\hline & 2 & 66.61 & 66.56 & 0.08 & \multirow[t]{2}{*}{$10^{7}$} & 2 & 73.83 & 73.58 & 0.34 \\
\hline & 3 & 73.18 & 72.55 & 0.86 & & 3 & 78.09 & 77.71 & 0.49 \\
\hline
\end{tabular}
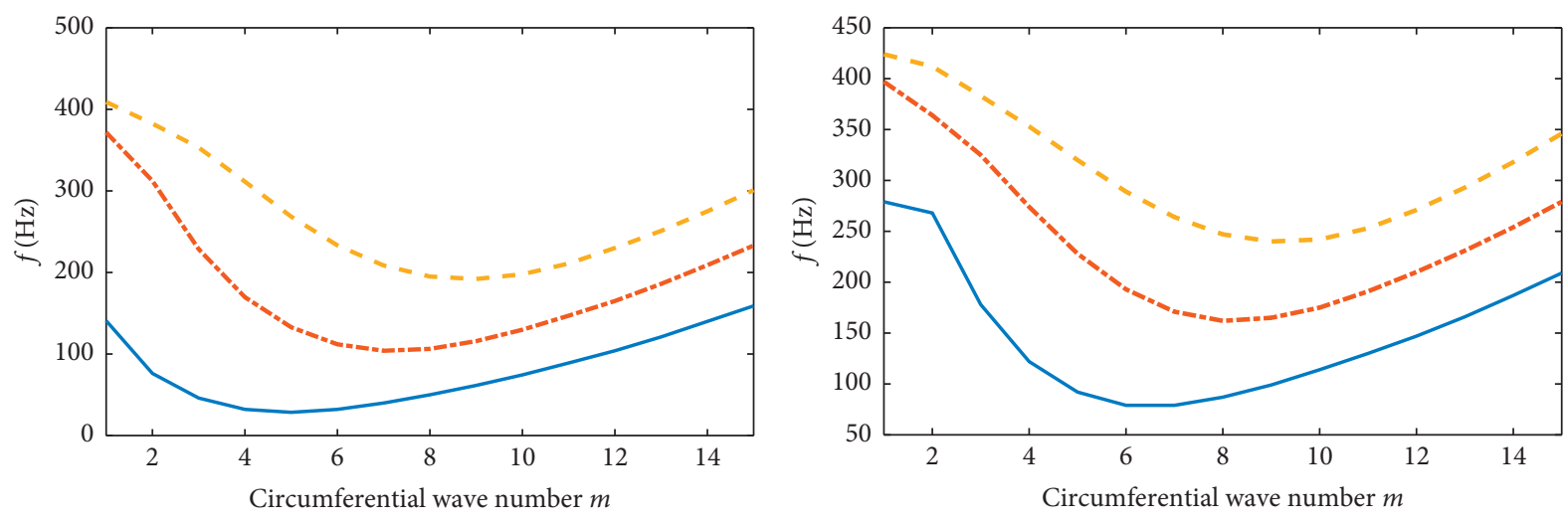

$$
\begin{array}{ll}
- & n=1 \\
- & n=2 \\
--- & n=3
\end{array}
$$

(a)

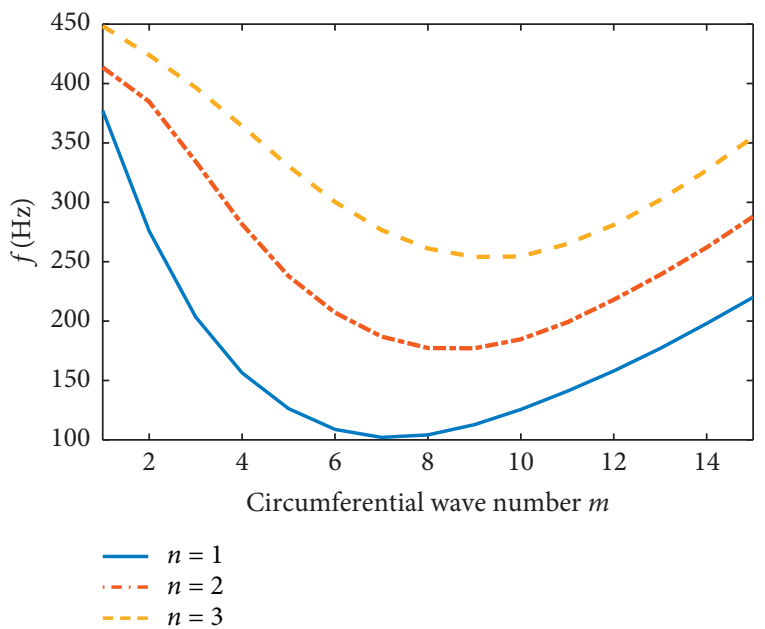

(c)

$$
\begin{array}{ll}
- & n=1 \\
-\cdots & n=2 \\
--- & n=3
\end{array}
$$

(b)

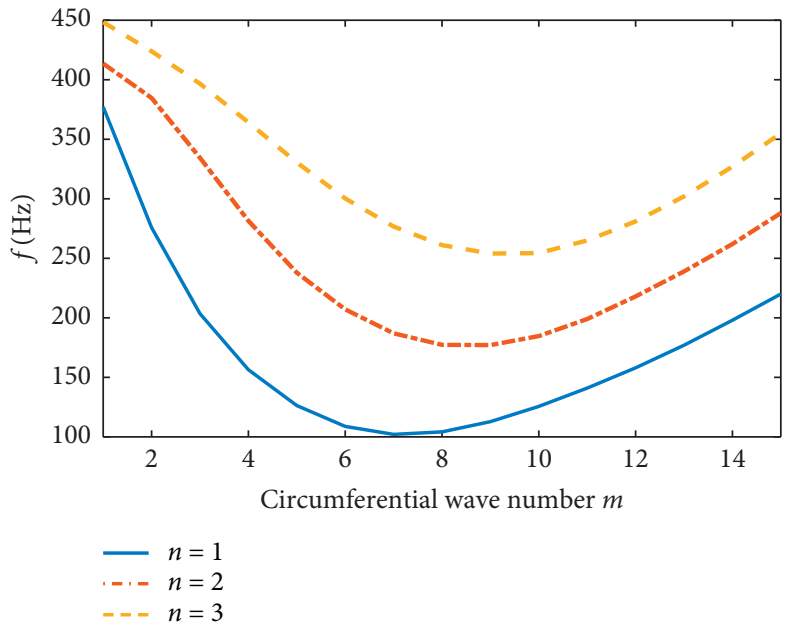

(d)

Figure 7: Frequency of conical under different boundary conditions, $m=1-3, n=1-15$. (a) C-F. (b) S-S. (c) S-C. (d) C-C.

Figure 12 shows the variation of the first 30 natural frequencies of the jointed conical shell. In can be seen that the trend of the curves in the four subgraphs is very similar. When the frequency order is very small, frequencies of model 2 are the largest and the frequencies of model 1 are the smallest. The values of the three curves are nearly the same when the mode number is 3 or 4 . Then, the frequency of model 2 becomes the smallest, which is significantly smaller than that of model 1 and model 3. At this point, frequencies of model 1 and model 3 become very close to each other; 
TABLE 4: Frequencies of conical shell with different half-cone angel $\alpha$

\begin{tabular}{|c|c|c|c|c|c|c|c|c|c|}
\hline \multirow{2}{*}{ Boundary condition } & \multirow{2}{*}{ Mode number } & \multicolumn{8}{|c|}{$f(\mathrm{~Hz})$} \\
\hline & & $10^{\circ}$ & $20^{\circ}$ & $30^{\circ}$ & $40^{\circ}$ & $50^{\circ}$ & $60^{\circ}$ & $70^{\circ}$ & $80^{\circ}$ \\
\hline \multirow{6}{*}{$\mathrm{C}-\mathrm{F}$} & 1 & 43.87 & 39.28 & 35.38 & 29.42 & 24.22 & 19.29 & 13.53 & 8.93 \\
\hline & 2 & 44.47 & 41.89 & 35.49 & 32.66 & 26.00 & 20.20 & 17.03 & 10.15 \\
\hline & 3 & 48.83 & 43.56 & 41.10 & 33.33 & 31.14 & 24.60 & 17.82 & 15.36 \\
\hline & 4 & 52.43 & 49.20 & 43.90 & 41.35 & 32.75 & 31.68 & 23.67 & 16.69 \\
\hline & 5 & 57.55 & 56.69 & 50.29 & 45.90 & 41.85 & 32.52 & 31.81 & 22.22 \\
\hline & 6 & 68.82 & 59.49 & 61.60 & 51.58 & 50.87 & 41.91 & 36.79 & 30.54 \\
\hline \multirow{6}{*}{ S-S } & 1 & 19.07 & 38.87 & 57.65 & 73.21 & 82.78 & 79.57 & 70.66 & 46.52 \\
\hline & 2 & 84.83 & 86.20 & 87.08 & 87.90 & 84.91 & 79.57 & 70.66 & 51.25 \\
\hline & 3 & 85.34 & 88.99 & 90.14 & 88.19 & 88.68 & 83.29 & 71.73 & 52.97 \\
\hline & 4 & 91.88 & 91.25 & 94.25 & 96.68 & 91.44 & 89.48 & 77.56 & 57.41 \\
\hline & 5 & 92.11 & 101.8 & 99.89 & 101.2 & 102.3 & 92.06 & 87.68 & 67.53 \\
\hline & 6 & 108.8 & 102.0 & 113.5 & 109.2 & 111.1 & 103.5 & 89.17 & 71.50 \\
\hline \multirow{6}{*}{$\mathrm{S}-\mathrm{C}$} & 1 & 98.28 & 102.2 & 106.0 & 106.5 & 104.9 & 99.26 & 84.63 & 60.15 \\
\hline & 2 & 100.4 & 104.4 & 106.2 & 109.7 & 108.4 & 99.83 & 86.67 & 61.23 \\
\hline & 3 & 102.2 & 107.7 & 113.3 & 112.3 & 112.1 & 106.8 & 90.13 & 66.80 \\
\hline & 4 & 109.4 & 112.6 & 116.0 & 123.3 & 117.5 & 111.6 & 100.0 & 75.74 \\
\hline & 5 & 126.1 & 122.0 & 125.4 & 125.2 & 129.8 & 117.6 & 103.5 & 77.76 \\
\hline & 6 & 151.8 & 146.1 & 137.0 & 137.3 & 134.8 & 131.2 & 113.2 & 91.65 \\
\hline \multirow{6}{*}{$\mathrm{C}-\mathrm{C}$} & 1 & 109.2 & 112.3 & 113.0 & 112.2 & 109.1 & 100.8 & 85.16 & 60.43 \\
\hline & 2 & 110.8 & 112.8 & 115.8 & 115.2 & 110.1 & 102.2 & 88.54 & 62.25 \\
\hline & 3 & 113.3 & 117.9 & 117.4 & 118.0 & 118.0 & 107.0 & 90.20 & 66.84 \\
\hline & 4 & 123.5 & 120.1 & 127.3 & 124.4 & 118.7 & 116.1 & 100.0 & 76.68 \\
\hline & 5 & 140.4 & 135.2 & 127.5 & 134.6 & 129.9 & 117.6 & 106.0 & 77.76 \\
\hline & 6 & 165.2 & 158.9 & 148.5 & 137.5 & 141.7 & 131.2 & 113.2 & 91.65 \\
\hline
\end{tabular}

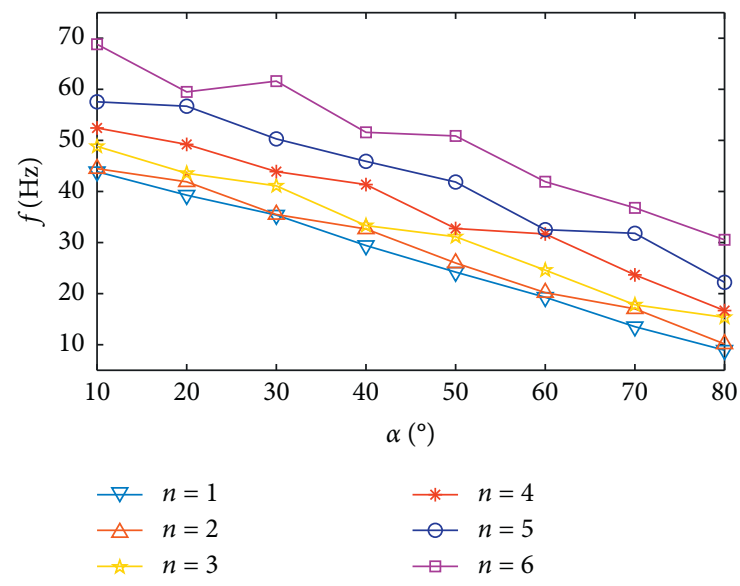

(a)

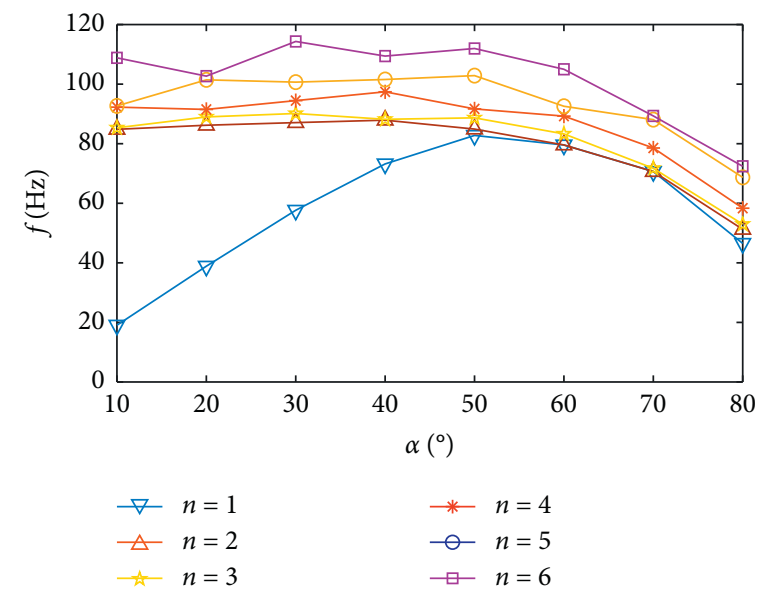

(b)

Figure 8: Continued. 


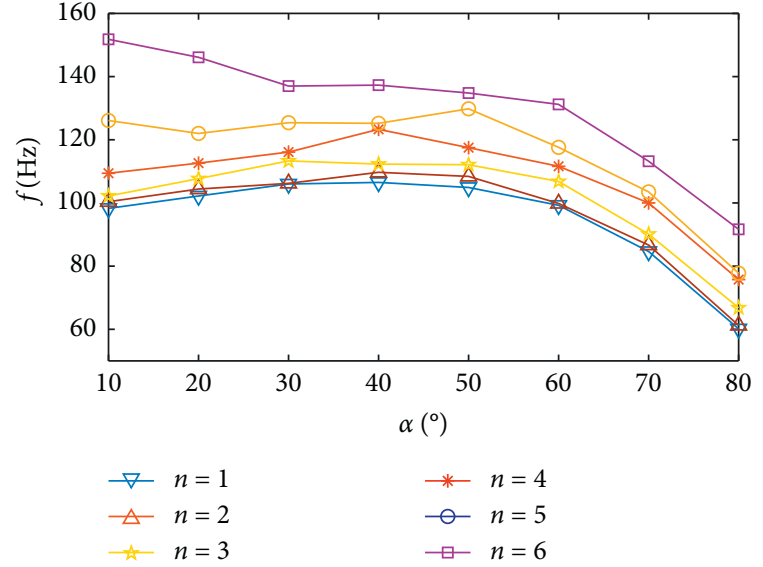

(c)

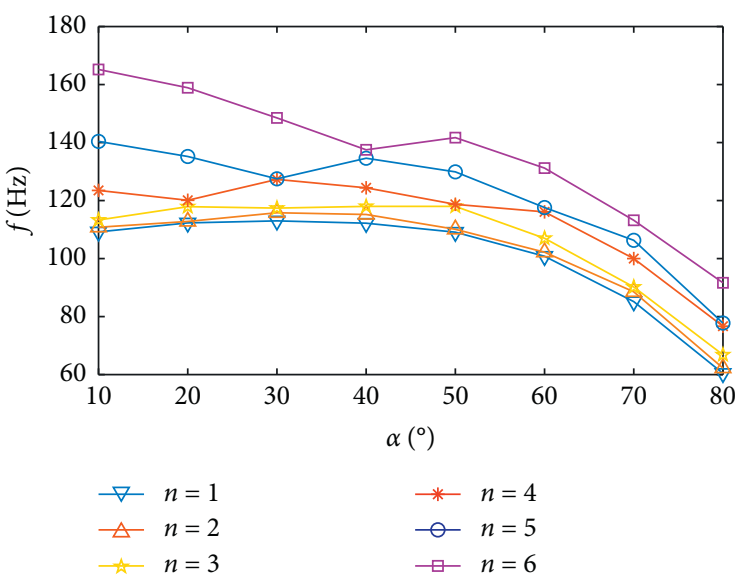

(d)

Figure 8: Frequency curves of the first six orders of conical shell with different half-cone angles. (a) C-F. (b) S-S. (c) S-C. (d) C-C.

TAble 5: Frequency of conical shell with different length-diameter ratios ( $\mathrm{Hz})$.

\begin{tabular}{|c|c|c|c|c|c|c|c|c|c|}
\hline \multirow{2}{*}{ Boundary condition } & \multirow{2}{*}{ Mode number } & \multicolumn{8}{|c|}{$L_{c}, \sin \alpha / R_{1}$} \\
\hline & & 0.1 & 0.2 & 0.3 & 0.4 & 0.5 & 0.6 & 0.7 & 0.8 \\
\hline \multirow{6}{*}{$\mathrm{C}-\mathrm{F}$} & 1 & 175.0 & 84.53 & 53.80 & 38.33 & 28.48 & 21.82 & 17.19 & 12.00 \\
\hline & 2 & 180.8 & 84.67 & 56.73 & 40.68 & 32.07 & 23.83 & 17.91 & 15.96 \\
\hline & 3 & 190.9 & 89.48 & 57.09 & 42.48 & 32.19 & 28.71 & 22.73 & 16.90 \\
\hline & 4 & 205.3 & 90.71 & 64.98 & 50.83 & 39.88 & 30.65 & 30.28 & 22.64 \\
\hline & 5 & 224.1 & 103.2 & 67.20 & 51.62 & 46.19 & 39.58 & 30.51 & 30.49 \\
\hline & 6 & 247.4 & 123.0 & 76.07 & 61.78 & 49.94 & 49.34 & 39.56 & 38.76 \\
\hline \multirow{6}{*}{$\mathrm{C}-\mathrm{C}$} & 1 & 472.9 & 215.1 & 150.2 & 119.8 & 102.4 & 92.24 & 85.48 & 81.90 \\
\hline & 2 & 474.4 & 219.7 & 151.3 & 121.9 & 104.5 & 93.01 & 87.61 & 84.66 \\
\hline & 3 & 477.8 & 228.9 & 155.1 & 125.0 & 109.3 & 100.7 & 91.29 & 89.46 \\
\hline & 4 & 482.9 & 243.1 & 166.4 & 130.0 & 113.0 & 102.7 & 100.5 & 91.27 \\
\hline & 5 & 489.5 & 262.4 & 184.7 & 138.5 & 125.6 & 111.9 & 105.0 & 100.5 \\
\hline & 6 & 497.0 & 287.0 & 210.9 & 161.2 & 126.8 & 125.4 & 111.9 & 112.0 \\
\hline \multirow{6}{*}{ S-S } & 1 & 108.3 & 76.12 & 64.66 & 58.85 & 56.07 & 55.36 & 56.00 & 57.59 \\
\hline & 2 & 256.5 & 175.6 & 120.1 & 94.05 & 78.61 & 68.33 & 61.27 & 58.79 \\
\hline & 3 & 342.8 & 181.9 & 122.8 & 94.32 & 78.71 & 69.93 & 67.21 & 60.73 \\
\hline & 4 & 347.1 & 194.8 & 124.8 & 101.5 & 86.95 & 75.95 & 67.30 & 67.47 \\
\hline & 5 & 353.4 & 214.2 & 134.4 & 105.3 & 91.56 & 86.90 & 76.26 & 73.10 \\
\hline & 6 & 361.1 & 239.7 & 156.2 & 114.0 & 99.24 & 88.77 & 86.99 & 76.28 \\
\hline \multirow{6}{*}{ S-C } & 1 & 398.7 & 196.3 & 138.1 & 111.1 & 95.84 & 86.89 & 82.90 & 81.30 \\
\hline & 2 & 401.4 & 201.3 & 141.1 & 114.1 & 99.81 & 91.01 & 84.33 & 84.65 \\
\hline & 3 & 406.2 & 211.8 & 141.8 & 115.9 & 101.0 & 94.01 & 91.21 & 85.03 \\
\hline & 4 & 412.7 & 228.0 & 153.0 & 126.4 & 111.7 & 100.3 & 96.73 & 91.27 \\
\hline & 5 & 420.3 & 250.2 & 172.1 & 126.6 & 115.9 & 111.9 & 100.5 & 100.5 \\
\hline & 6 & 428.5 & 278.0 & 200.2 & 149.6 & 125.3 & 117.6 & 111.9 & 112.0 \\
\hline
\end{tabular}

strictly speaking, the frequency value of model 1 is slightly higher on the whole. The influence of boundary conditions on the natural frequency values of the three sets of models is mainly reflected in the sequence of the natural frequency values of the lower orders and the mode number corresponding to the intersection point of the three curves. In summary, increasing the thickness of the conical shell in the larger radius end and decreasing the thickness of the conical shell in the smaller radius end will reduce the natural frequency values of the first few orders of the structure, while slightly increasing the frequency values in the higher-order range. Increasing the thickness of the conical shell in smaller radius end and decreasing the thickness of the conical shell in the larger radius end will increase the natural frequency values of the first few orders and greatly reduce the frequency values in the higher-order range.

Figure 13 shows the comparison of the first six modes of the stepped joined conical shells under $\mathrm{C}-\mathrm{C}$ boundary condition. Using the mode shape of Model 3 as a reference, it is apparently that the vibration area of Model 1 is more inclined to the end with a small radius, while the vibration area of Model 2 is more inclined to the end with a larger 

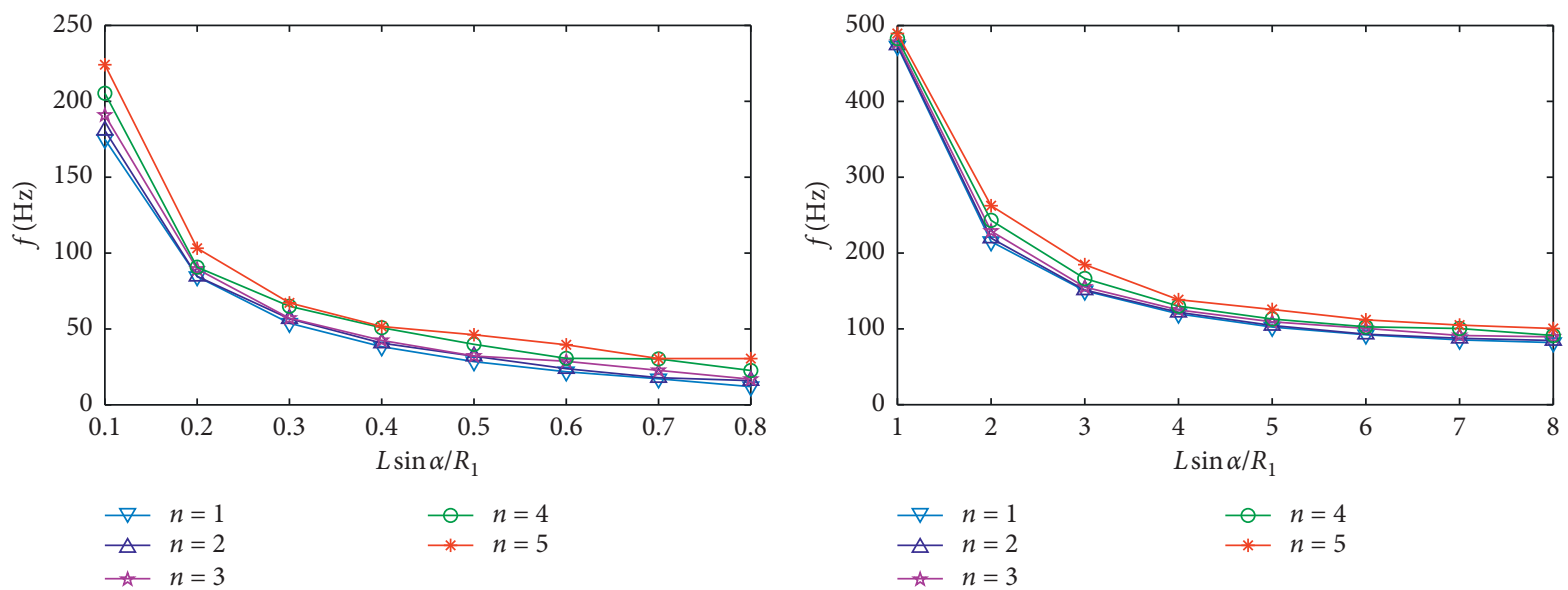

$\begin{array}{ll}\triangle & n=1 \\ \triangle & n=2 \\ \text { त } & n=3\end{array}$

$\begin{array}{ll}- & n=4 \\ - & n=5\end{array}$

(a)

(b)
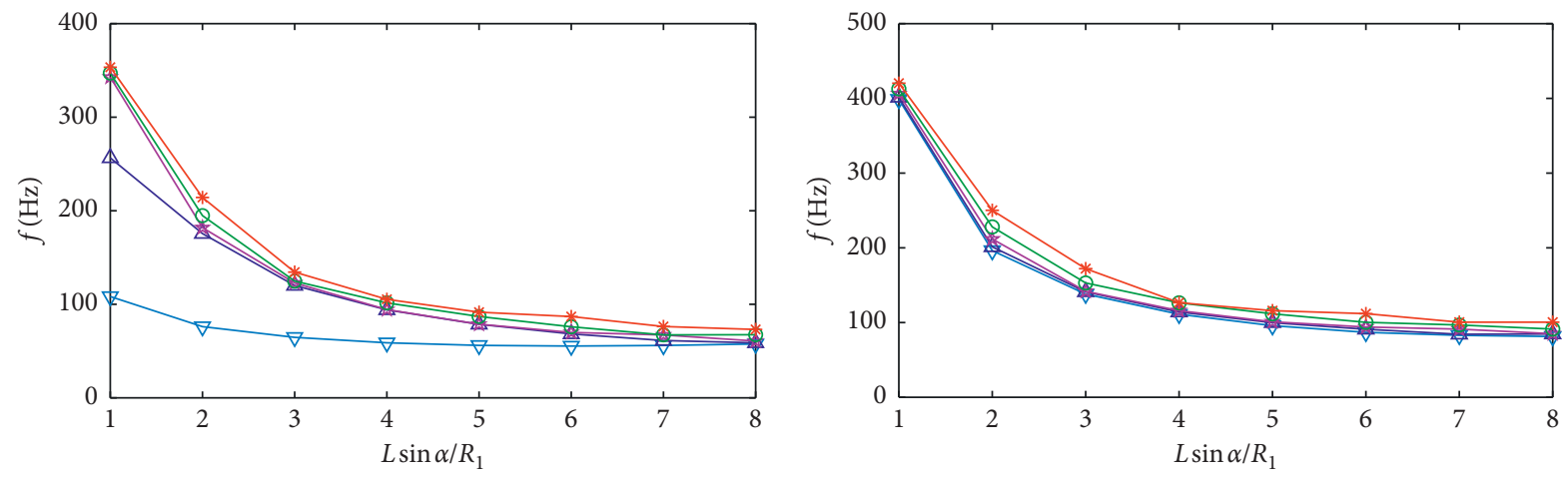

$\nabla n=1$
$\triangle n=2$
^ $n=3$

○- $n=4$

$\nabla n=1$

$\multimap n=4$

$\triangle n=2$

$\rightarrow n=5$

त $n=3$

(c)

(d)

FiguRE 9: Frequency curves of the first 3 orders of the conical shell with different length-diameter ratios. (a) C-F. (b) C-C. (c) S-S. (d) S-C.

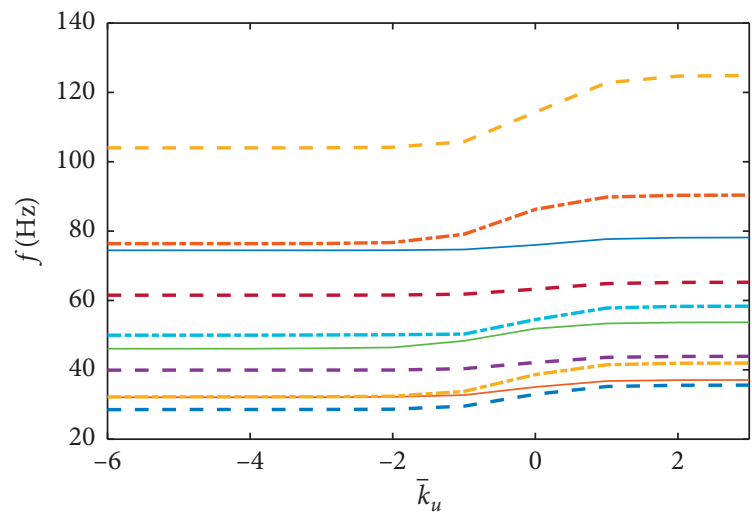

(a)

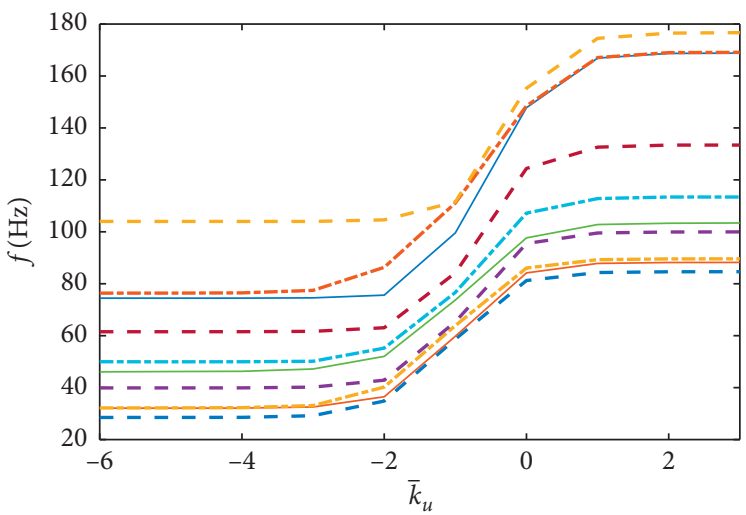

(b)

FIGURE 10: Continued. 


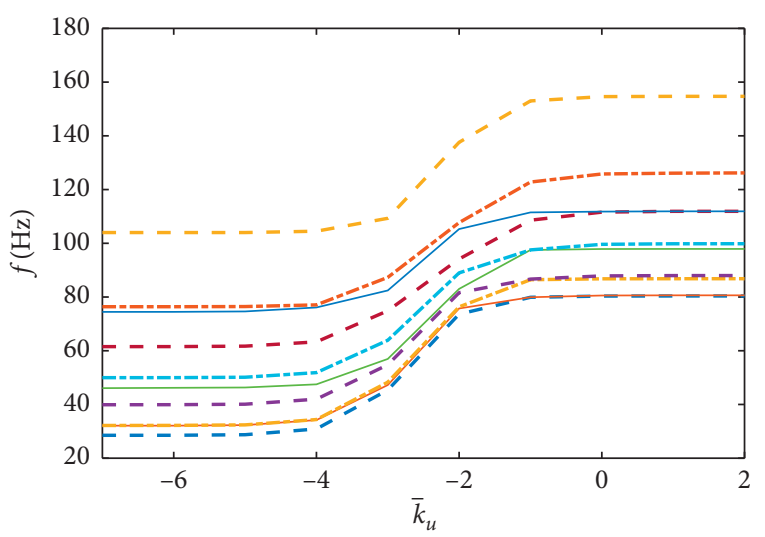

(c)

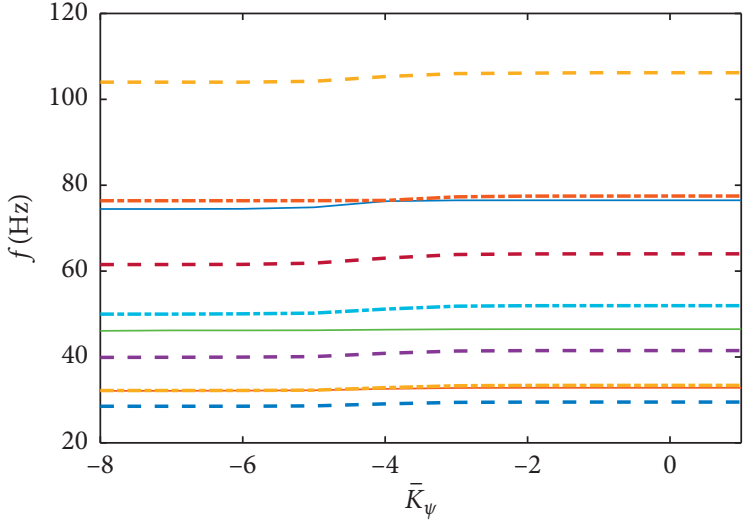

(d)

Figure 10: Frequency curves of the first 10 orders of conical shell with one displacement elastically restrained and the others free.

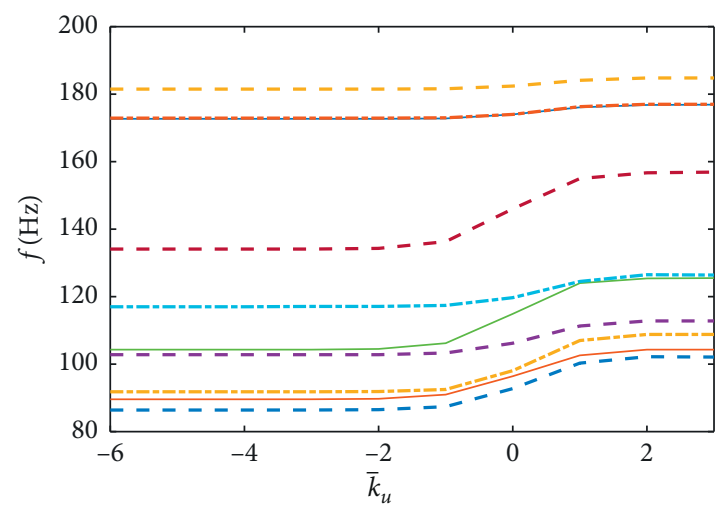

(a)

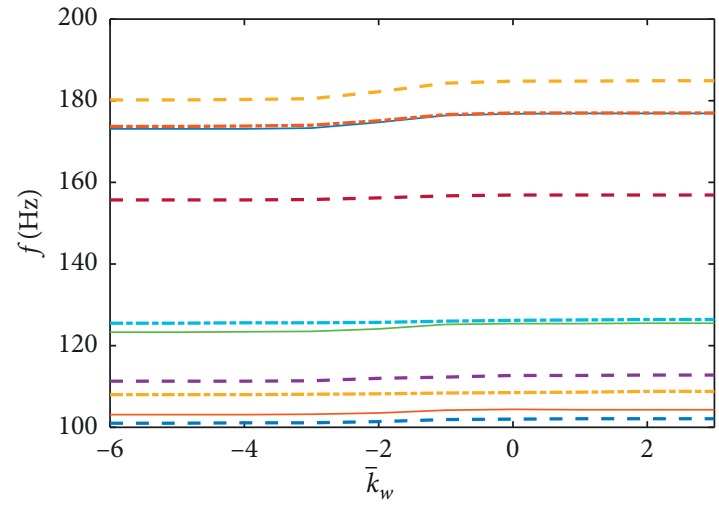

(c)

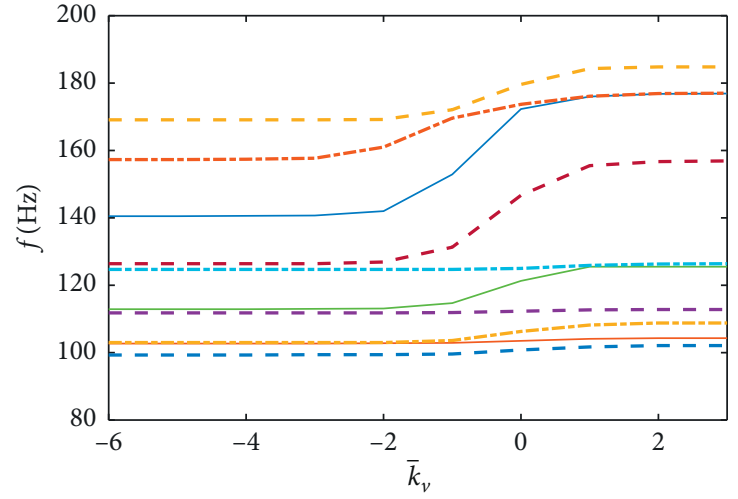

(b)

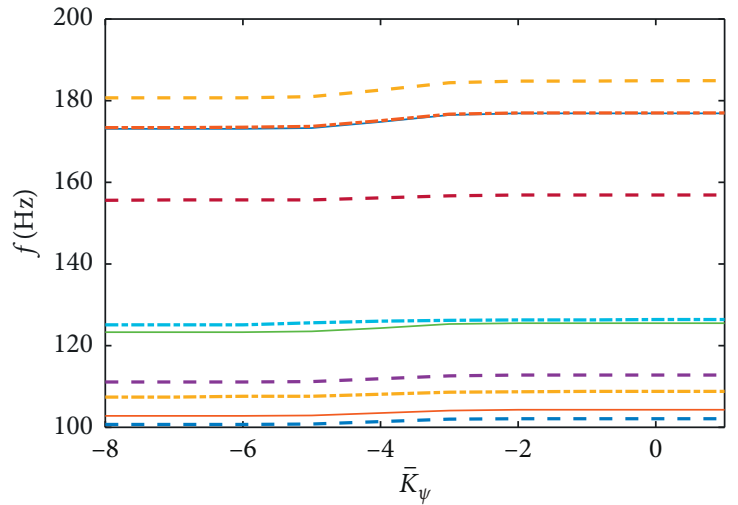

(d)

FIGURE 11: Frequency curves of the first 10 orders of conical shell with one displacement elastically restrained and the others fixed.

radius. This is mainly due to the reduction of the thickness of the conical shell at the smaller radius in Model 1 and the reduction of the thickness of the conical shell at the larger radius in Model 2. Therefore, the vibration mode of the joint shell can be controlled by adjusting the thickness at different position.

Next, free vibration analysis of joint conical shell with different semivertex angle is performed. In the numerical examples, three sets of joint conical shell made of two shell with different semivertex angle are considered: (1) model $1, \alpha_{1}=\pi / 3$ and $\alpha_{2}=\pi / 3$; (2) model 2, $\alpha_{1}=\pi / 3$ and $\alpha_{2}=\pi / 4$; (3) model $3, \alpha_{1}=\pi / 3$ and $\alpha_{2}=\pi / 6$. The lengths of the two subconical shells are $1 \mathrm{~m}$, and the average radius of the first shell is $R_{0}=0.933 \mathrm{~m}$. In Figure 14, the first 30 frequencies of the three joint conical shells under $\mathrm{C}-\mathrm{C}$, $\mathrm{S}-\mathrm{S}, \mathrm{F}-\mathrm{F}$, and $\mathrm{C}-\mathrm{F}$ boundary conditions are given, 


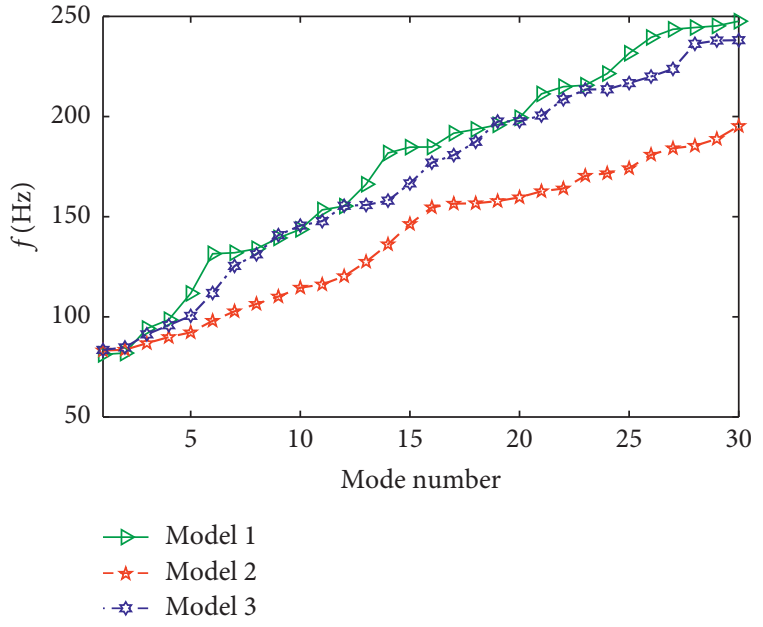

(a)

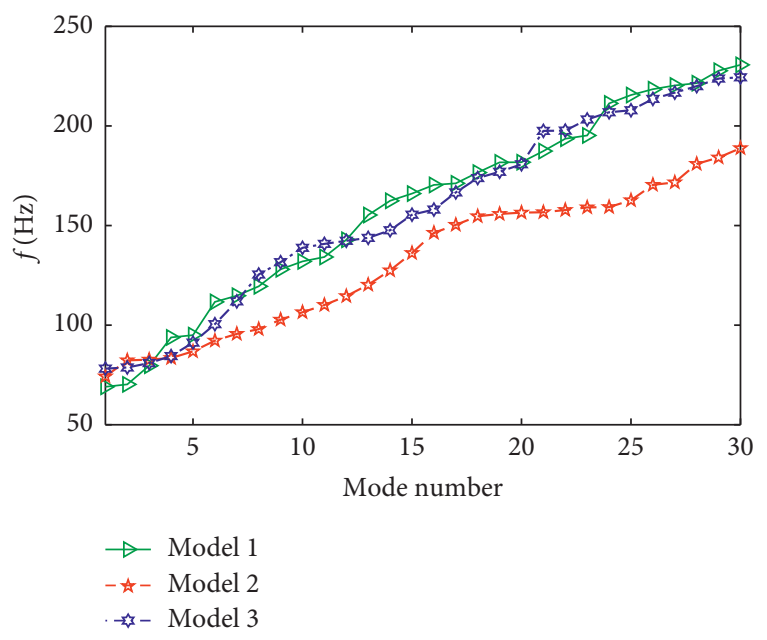

(c)

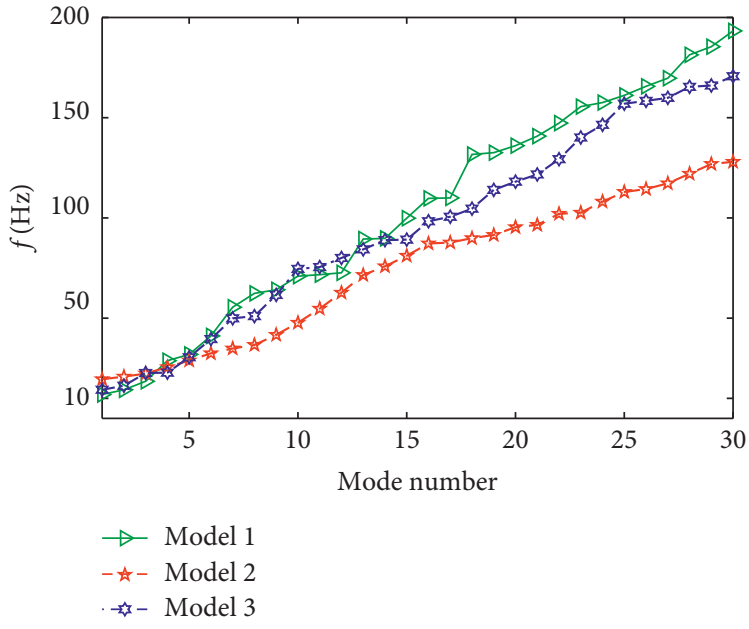

(b)

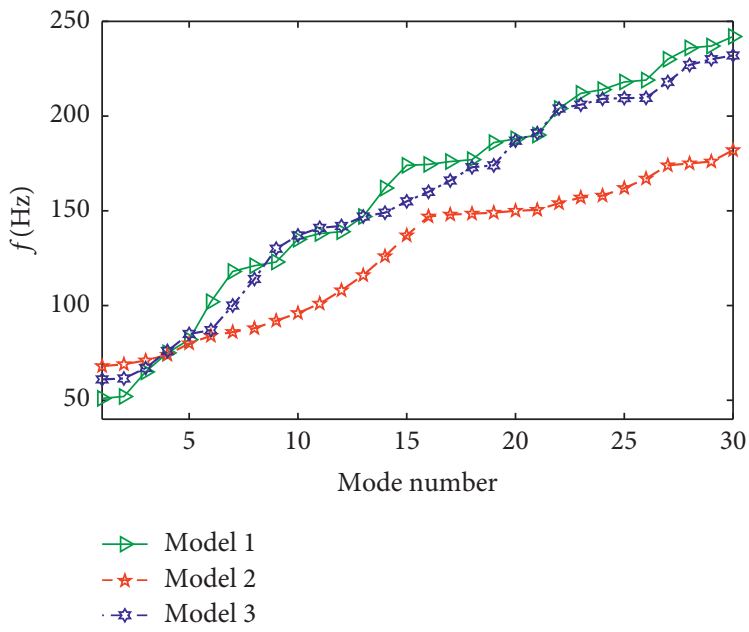

(d)

Figure 12: First 30 orders of frequencies of stepped joined conical shells under different boundary conditions.

respectively (the first letter represents the boundary condition in the small radius end and the second letter represents the boundary condition in the large radius end). It is observed that the curves in Figures 14(a) and 14(b) are very similar to each other in the aspects of tendence and amplitude, which means that the change of boundary condition from $\mathrm{C}-\mathrm{C}$ to $\mathrm{S}-\mathrm{S}$ has little effect on natural frequency. By comparing Figures 14(a) and 14(d), one can see that the natural frequency significantly decreases as the boundary changes from $\mathrm{C}-\mathrm{C}$ to $\mathrm{C}-\mathrm{F}$, especially in lower frequency range. When the boundary restraint is further released from $\mathrm{C}-\mathrm{F}$ to $\mathrm{F}-\mathrm{F}$, the change of frequency in Figures 14(c) and 14(d) is not evident. It is concluded that the boundary constraint in the larger radius end has greater impact on the vibration characteristics of the joined conical shell. It is evident that an increase of $\alpha_{2}$ leads to an increase of frequency of the joined conical shell. This is mainly due to the decrease of the semivertex angle, which directly results in the decrease of the average radius of the shell and the increase of the stiffness at the same time. However, it is worth noting that the differences of the first 10 frequencies in the curves of Figures 14(c) and 14(d) are almost negligible and after the $10^{\text {th }}$ order, the differences gradually appear. The reason for the phenomenon might be due to the free boundary restraint.

In the last numerical example, it is assumed that one of the semivertex angle of the subconical shell is $90^{\circ}$. Then, the joined shell can be regarded as a conical shell coupled with an annular plate. In the example, the geometric parameters are as follows: $R_{0}=1 \mathrm{~m}, L=2 \mathrm{~m}$, and $\alpha=\pi / 6$ for the conical shell, and $R_{\text {in }}=1 \mathrm{~m}$ and $R_{\text {out }}=1.5 \mathrm{~m}$ for the annular plate, and $h=0.01 \mathrm{~m}$. The outer boundary line of the annular plate is coupled to the large radius end of the conical shell. Table 6 shows the comparison of the natural frequency for the joined shell obtained by the present method and the FEM. The FEM software ANSYS is applied, in which Shell 63 element is chosen and the mesh number is $300 \times 160$. It is seen that the present results agree well with those obtained from ANSYS. Thus, it can be demonstrated that the present method is qualified for free vibration analysis of conical shell-annular plate coupled structures. 

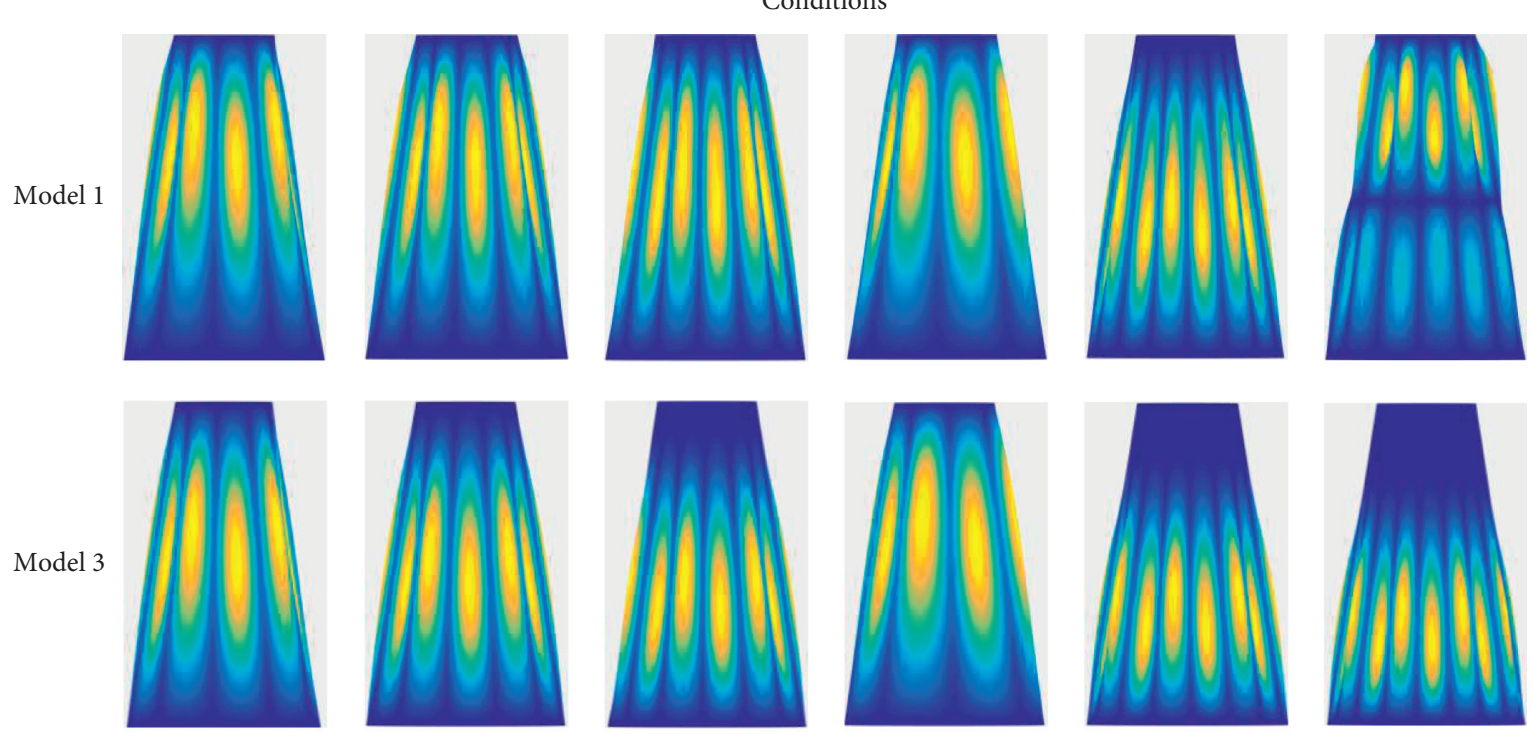

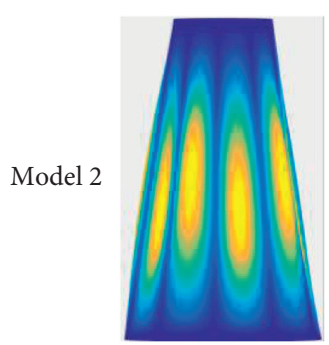

$1^{\text {st }}$

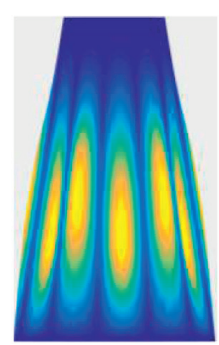

$2^{\text {nd }}$

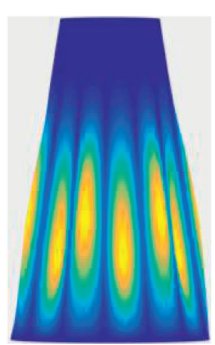

$3^{\text {rd }}$

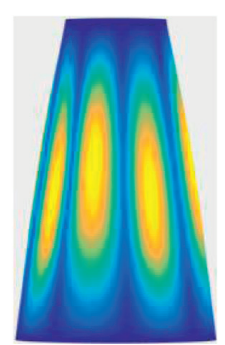

$4^{\text {th }}$

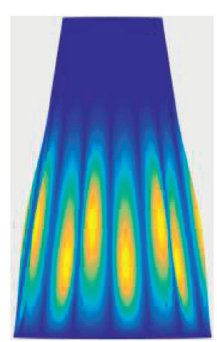

$5^{\text {th }}$

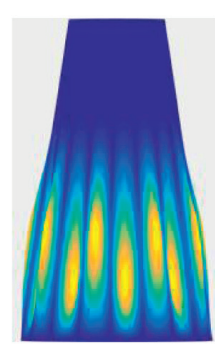

$6^{\text {th }}$

Figure 13: Mode shapes of stepped joined conical shell under C-C boundary condition.
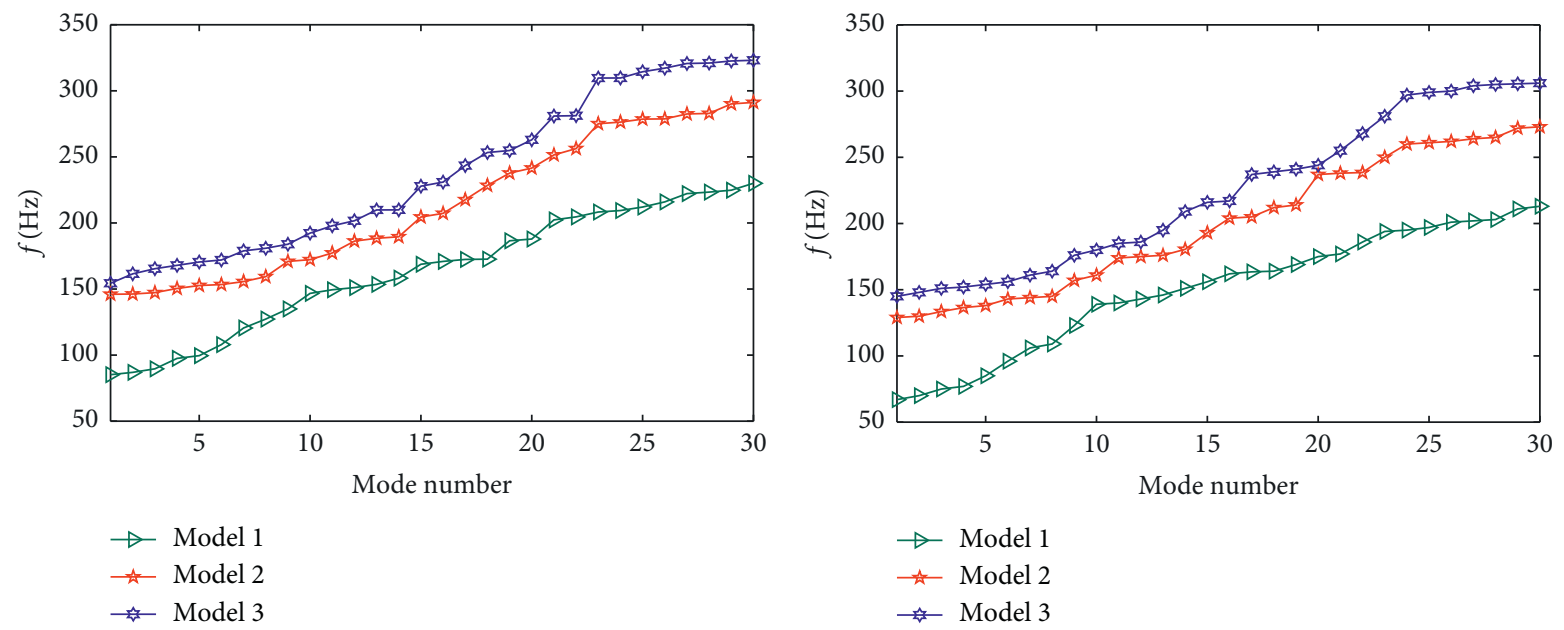

(a)

(b)

Figure 14: Continued. 


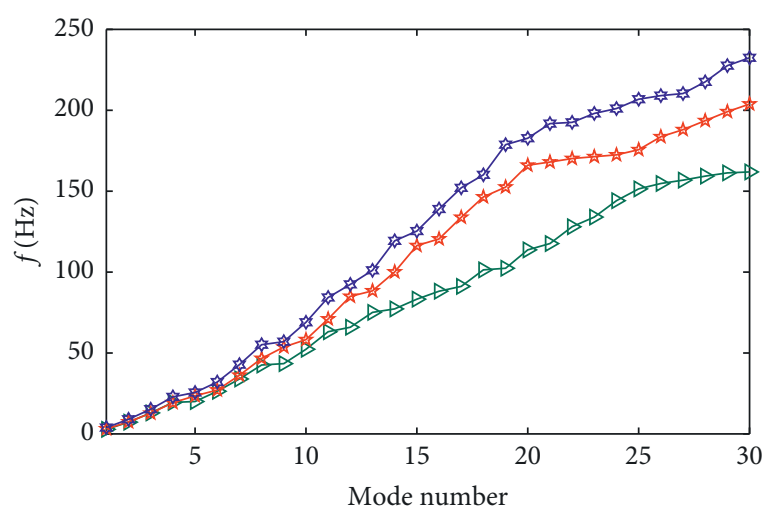

$\rightarrow$ Model 1
$\rightarrow$ Model 2
$\rightarrow$ Model 3

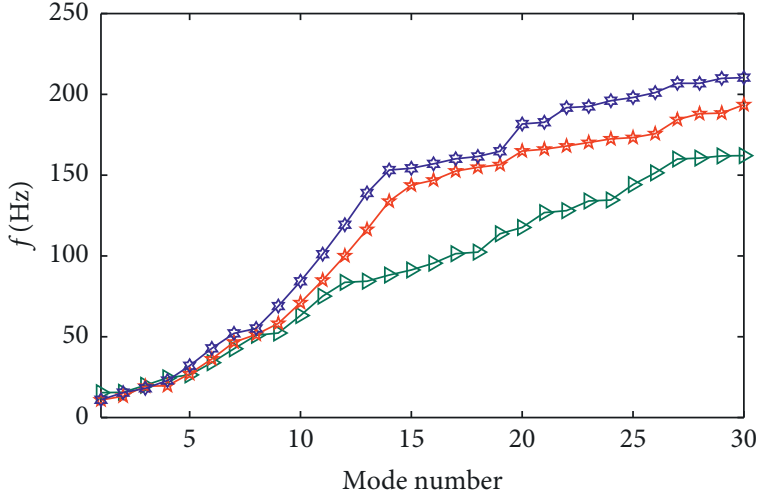

$\rightarrow$ Model 1

$\star$ Model 2

\# Model 3

(c)

(d)

FIGURE 14: Frequency curves of the first 30 orders of three conical shells with variable cone angle under different boundary conditions. (a) C-C. (b) S-S. (c) F-F. (d) C-F.

TABLE 6: Frequencies of conical shell-annular plate structure under different boundary conditions.

\begin{tabular}{|c|c|c|c|c|c|c|c|c|c|}
\hline \multirow{2}{*}{ Boundary condition } & \multirow{2}{*}{$m$} & \multirow{2}{*}{ Method } & \multicolumn{7}{|c|}{$f(\mathrm{~Hz})$} \\
\hline & & & 1 & 2 & 3 & 4 & 5 & 6 & 7 \\
\hline \multirow{8}{*}{ F-F } & \multirow{2}{*}{1} & Present & 38.68 & 207.7 & 476.3 & 539.1 & 556.3 & 608.9 & 658.5 \\
\hline & & FEM & 38.15 & 207.6 & 477.2 & 539.1 & 556.9 & 608.9 & 658.3 \\
\hline & \multirow{2}{*}{2} & Present & 16.76 & 43.44 & 152.1 & 211.1 & 359.7 & 484.3 & 544.3 \\
\hline & & FEM & 17.84 & 43.45 & 152.6 & 211.5 & 360.3 & 484.8 & 544.9 \\
\hline & \multirow{2}{*}{3} & Present & 44.39 & 54.64 & 210.8 & 236.4 & 379.2 & 473.6 & 511.4 \\
\hline & & FEM & 44.80 & 54.78 & 211.3 & 237.0 & 379.8 & 474.6 & 512.4 \\
\hline & \multirow{2}{*}{4} & Present & 69.03 & 75.31 & 180.3 & 232.1 & 316.7 & 428.8 & 516.7 \\
\hline & & FEM & 69.18 & 75.57 & 181.1 & 232.6 & 316.9 & 429.4 & 517.0 \\
\hline \multirow{8}{*}{$\mathrm{C}-\mathrm{C}$} & \multirow{2}{*}{1} & Present & 191.8 & 348.9 & 454.6 & 557.4 & 575.9 & 626.2 & 669.1 \\
\hline & & FEM & 192.2 & 349.5 & 455.2 & 557.3 & 576.4 & 626.3 & 669.4 \\
\hline & \multirow{2}{*}{2} & Present & 193.6 & 237.3 & 386.3 & 520.5 & 561.1 & 613.2 & 673.8 \\
\hline & & FEM & 194.0 & 238.0 & 386.4 & 521.1 & 561.1 & 613.0 & 673.2 \\
\hline & \multirow{2}{*}{3} & Present & 154.3 & 198.3 & 327.3 & 455.2 & 530.4 & 574.1 & 629.6 \\
\hline & & FEM & 154.8 & 198.6 & 327.6 & 455.6 & 530.9 & 574.5 & 629.2 \\
\hline & \multirow{2}{*}{4} & Present & 113.2 & 203.8 & 270.7 & 392.7 & 487.6 & 550.8 & 595.2 \\
\hline & & FEM & 113.5 & 204.2 & 271.1 & 393.0 & 487.7 & 551.1 & 695.1 \\
\hline
\end{tabular}

\section{Conclusion}

In this paper, an efficient modeling technique based on the dynamic stiffness method has been formulated to study the free vibration characteristics of conical shell and its combinations with uniform boundary restraints. Based on the Flügge thin shell theory, the general solutions are expressed in a power series form. Then, by means of the governing equation, the general solutions are replaced by a simplified form which contains eight equivalent shape functions. Giving the displacements and forces on the boundaries in vector form and eliminating the integration constants, the dynamic stiffness matrix is formulated. The elastic boundary restraint is applied by introducing four sets of boundary springs at the ends of the conical shell, which make it capable of coping with arbitrarily prescribed boundary conditions. Excellent convergence and accuracy of the present formulation have been examined through numerical examples and comparison of the present results with those of other methods. The effects of half-cone angle, length-diameter ratio, and elastic boundary restraints on the vibration characteristics of the conical shell are studied. Investigation of geometric property on vibration behavior on joined conical shell is also conducted. Although the present study is only focused on free vibration of conical shell, the present formulation can be conveniently converted to forced vibration analysis. As to the forced vibration, the 
dynamic load is comprised in the force vector $\mathbf{Q}_{m}$ and the dynamic response is obtained by solving equation of motion in equation (21).

\section{Data Availability}

The data used to support the findings of this study are available from the corresponding author upon request.

\section{Acknowledgments}

This research was funded by the National Natural Science Foundation of China (Grant numbers: 51822902 and 51775125).

\section{Appendix.}

\section{Conflicts of Interest}

The authors declare that they have no conflicts of interest.

$$
\begin{aligned}
& L_{11}=-\frac{s^{2}}{R^{2}}+\frac{s}{R} \frac{\partial}{\partial x}+\frac{\partial^{2}}{\partial x^{2}}+\frac{1-v}{2 R^{2}} \frac{\partial^{2}}{\partial \theta^{2}}+k\left(\frac{1-v}{2 R^{2}} \frac{c^{2}}{R^{2}} \frac{\partial^{2}}{\partial \theta^{2}}-\frac{c^{2} s^{2}}{R^{4}}\right)-\rho h \frac{\partial^{2}}{\partial t^{2}}, \\
& L_{12}=\frac{1+v}{2 R} \frac{\partial^{2}}{\partial x \partial \theta}-\frac{3-v}{2} \frac{s}{R^{2}} \frac{\partial}{\partial \theta} \\
& L_{13}=\frac{v c}{R} \frac{\partial}{\partial x}-\frac{s c}{R^{2}}-k\left(\frac{s c^{3}}{R^{4}}+\frac{s^{2} c}{R^{3}} \frac{\partial}{\partial x}+\frac{c}{R} \frac{\partial^{3}}{\partial x^{3}}-\frac{(1-v) c}{2 R^{3}} \frac{\partial^{3}}{\partial x \partial \theta^{2}}+\frac{(3-v) s c}{2 R^{4}} \frac{\partial^{2}}{\partial \theta^{2}}\right), \\
& L_{21}=\frac{3-v}{2} \frac{s}{R^{2}} \frac{\partial}{\partial \theta}+\frac{1+v}{2 R} \frac{\partial^{2}}{\partial x \partial \theta} \\
& L_{22}=\frac{1}{R^{2}} \frac{\partial^{2}}{\partial \theta^{2}}-\frac{1-v}{2} \frac{s^{2}}{R^{2}}+\frac{1-v}{2} \frac{s}{R} \frac{\partial}{\partial x}+\frac{1-v}{2} \frac{\partial^{2}}{\partial x^{2}} \\
& +k\left(\frac{3 s^{2} c^{2}(1-v)}{2 R^{4}}-\frac{(1-v) s c^{2}}{R^{3}} \frac{\partial}{\partial x}+\frac{3 c^{2}(1-v)}{2 R^{2}} \frac{\partial^{2}}{\partial x^{2}}\right)-\rho h \frac{\partial^{2}}{\partial t^{2}}, \\
& L_{23}=\frac{\cos \alpha}{R^{2}} \frac{\partial}{\partial \theta}-k\left[\frac{(1-v) s^{2} c}{R^{4}} \frac{\partial}{\partial \theta}-\frac{3(1-v) s c}{2 R^{3}} \frac{\partial^{2}}{\partial x \partial \theta}+\frac{c(3-v)}{2 R^{2}} \frac{\partial^{3}}{\partial x^{2} \partial \theta}\right], \\
& L_{31}=-\frac{s c}{R^{2}}-\frac{v c}{R} \frac{\partial}{\partial x}-k\left[\frac{2 s^{3} c}{R^{4}}-\frac{s^{2} c}{R^{3}} \frac{\partial}{\partial x}+\frac{s c^{3}}{R^{4}}-\frac{c}{R} \frac{\partial^{3}}{\partial x^{3}}+\frac{s c(1+v)}{2 R^{4}} \frac{\partial^{2}}{\partial \theta^{2}}+\frac{c(1+v)}{2 R^{3}} \frac{\partial^{2}}{\partial x \partial \theta^{2}}\right], \\
& L_{32}=-\frac{\cos \alpha}{R^{2}} \frac{\partial}{\partial \theta}-k\left[\frac{s c(3+v)}{2 R^{3}} \frac{\partial^{2}}{\partial x \partial \theta}-\frac{s^{2} c(3+v)}{2 R^{4}} \frac{\partial}{\partial \theta}-\frac{c(3-v)}{2 R^{2}} \frac{\partial^{3}}{\partial x^{2} \partial \theta}\right], \\
& L_{33}=-\frac{c^{2}}{R^{2}}-k \nabla^{4}-k \frac{c^{2}}{R^{4}}\left(2+c^{2}+2 \frac{\partial^{2}}{\partial \theta^{2}}\right)-\rho h \frac{\partial^{2}}{\partial t^{2}},
\end{aligned}
$$

where $\nabla^{4}=\nabla^{2} \nabla^{2}, \nabla^{2}=\left(\partial^{2} / \partial x^{2}\right)+(s / R)(\partial / \partial x)+\left(1 / R^{2}\right)\left(\partial^{2}\right.$ $\left./ \partial \theta^{2}\right), k=\left(h^{2} / 12\right)$.

\section{References}

[1] A. W. Leissa, Vibration of ShellsOhio State University Columbus, Columbus, OH, USA, 1973.

[2] R. D. Blevins, Formulas for Natural Frequencies and Mode Shapes, Krieger Publishing Company, Malabar, FL, USA, 2001.

[3] M. S. Qatu, "Recent research advances in the dynamic behavior of shells: 1989-2000, part 2: homogeneous shells," Applied Mechanics Reviews, vol. 55, no. 5, pp. 415-434, 2002.
[4] S. K. Sen and P. L. Gould, "Free vibration of shells of revolution using FEM," Journal of the Engineering Mechanics Division, vol. 100, pp. 283-303, 1974.

[5] K. R. Sivadas and N. Ganesan, "Vibration analysis of thick composite clamped conical shells of varying thickness," Journal of Sound and Vibration, vol. 152, no. 1, pp. 27-37, 1992.

[6] D. P. Thambiratnam and Y. Zhuge, "Axisymmetric free vibration analysis of conical shells," Engineering Structures, vol. 15, no. 2, pp. 83-89, 1993.

[7] T. Irie, G. Yamada, and K. Tanaka, "Natural frequencies of truncated conical shells," Journal of Sound and Vibration, vol. 92, no. 3, pp. 447-453, 1984. 
[8] V. Q. Hien, T. I. Thinh, N. M. Cuong, and P. N. Thanh, "Free vibration analysis of joined composite conical-conical-conical shells containing fluid," Vietnam Journal of Science and Technology, vol. 54, no. 5, pp. 650-663, 2016.

[9] X. Wang, W. Wu, and X. Yao, "Structural and acoustic response of a finite stiffened conical shell," Acta Mechanica Solida Sinica, vol. 28, no. 2, pp. 200-209, 2015.

[10] L. Tong, "Free vibration of orthotropic conical shells," International Journal of Engineering Science, vol. 31, no. 5, pp. 719-733, 1993.

[11] L. Tong, "Free vibration of composite laminated conical shells," International Journal of Mechanical Sciences, vol. 35, no. 1, pp. 47-61, 1993.

[12] Y. Kerboua, A. A. Lakis, and M. Hmila, "Vibration analysis of truncated conical shells subjected to flowing fluid," Applied Mathematical Modelling, vol. 34, no. 3, pp. 791-809, 2010.

[13] C. Shu, "An efficient approach for free vibration analysis of conical shells," International Journal of Mechanical Sciences, vol. 38, no. 8-9, pp. 935-949, 1996.

[14] H. Bagheri, Y. Kiani, and M. R. Eslami, "Free vibration of joined conical-conical shells," Thin-walled Structures, vol. 120, pp. 446-457, 2017.

[15] G. Ni and S. J. Elliott, "Wave interpretation of numerical results for the vibration in thin conical shells," Journal of Sound and Vibration, vol. 333, no. 10, pp. 2750-2758, 2014.

[16] Ö. Civalek, "Discrete singular convolution method for the free vibration analysis of rotating shells with different material properties," Composite Structures, vol. 160, pp. 267-279, 2017.

[17] R. Ansari, M. Faghih Shojaei, H. Rouhi, and M. Hosseinzadeh, "A novel variational numerical method for analyzing the free vibration of composite conical shells," Applied Mathematical Modelling, vol. 39, no. 10-11, pp. 2849-2860, 2015.

[18] A. J. Stanley and N. Ganesan, "Frequency response of shellplate combinations," Computers \& Structures, vol. 59, no. 6, pp. 1083-1094, 1996.

[19] E. Efraim and M. Eisenberger, "Exact vibration frequencies of segmented axisymmetric shells," Thin-walled Structures, vol. 44, no. 3, pp. 281-289, 2006.

[20] K. Xie, M. Chen, and Z. Li, "An analytic method for free and forced vibration analysis of stepped conical shells with arbitrary boundary conditions," Thin-walled Structures, vol. 111, pp. 126-137, 2017.

[21] M. Shakouri and M. A. Kouchakzadeh, "Free vibration analysis of joined conical shells: analytical and experimental study," Thin-Walled Structures, vol. 85, pp. 350-358, 2014.

[22] Y. G. Qu, Y. Chen, Y. F. Chen, X. H. Long, H. X. Hua, and G. Meng, "A domain decomposition method for vibration analysis of conical shells with uniform and stepped thickness," Journal of Vibration and Acoustics-Transactions of The Asme, vol. 135, Article ID 011014, 2013.

[23] G. Jin, X. Ma, S. Shi, T. Ye, and Z. Liu, "A modified Fourier series solution for vibration analysis of truncated conical shells with general boundary conditions," Applied Acoustics, vol. 85, pp. 82-96, 2014.

[24] Z. Song, Q. Cao, and Q. Dai, "Free vibration of truncated conical shells with elastic boundary constraints and added mass," International Journal of Mechanical Sciences, vol. 155, pp. 286-294, 2019.

[25] J. R. Banerjee, "The dynamic stiffness method: theory, practice and promise," Computational Technology Reviews, vol. 11, pp. 31-57, 2015.

[26] J. R. Banerjee and A. Ananthapuvirajah, "Free vibration of functionally graded beams and frameworks using the dynamic stiffness method," Journal of Sound and Vibration, vol. 422, pp. 34-47, 2018.

[27] J. $\mathrm{Li}, \mathrm{X} . \mathrm{Hu}$, and $\mathrm{X} . \mathrm{Li}$, "Free vibration analyses of axially loaded laminated composite beams using a unified higherorder shear deformation theory and dynamic stiffness method," Composite Structures, vol. 158, pp. 308-322, 2016.

[28] C. Zhang, G. Jin, T. Ye, and Y. Zhang, "Harmonic response analysis of coupled plate structures using the dynamic stiffness method," Thin-walled Structures, vol. 127, pp. 402-415, 2018.

[29] R. S. Langley, "A dynamic stiffness technique for the vibration analysis of stiffened shell structures," Journal of Sound and Vibration, vol. 156, no. 3, pp. 521-540, 1992.

[30] A. N. Bergin, "Natural frequencies of cross-ply laminated singly curved panels," Mechanics Research Communications, vol. 23, no. 2, pp. 165-170, 1996.

[31] N. Kolarevic, M. Nefovska-Danilovic, and M. Petronijevic, "Dynamic stiffness method in the vibration analysis of circular cylindrical shell," Gradjevinski Materijali I Konstrukcije, vol. 59, no. 3, pp. 45-61, 2016.

[32] M. A. Mahmoud, "Natural frequency of axially functionally graded, tapered cantilever beams with tip masses," Engineering Structures, vol. 187, pp. 34-42, 2019.

[33] J. R. Banerjee and A. Ananthapuvirajah, "Free flexural vibration of tapered beams," Computers \& Structures, vol. 224, Article ID 106106, 2019.

[34] J. R. Banerjee, A. Ananthapuvirajah, and S. O. Papkov, "Dynamic stiffness matrix of a conical bar using the RayleighLove theory with applications," European Journal of Mechanics - A/Solids, vol. 83, Article ID 104020, 2020.

[35] J. R. Banerjee, A. Ananthapuvirajah, X. Liu, and C. Sun, "Coupled axial-bending dynamic stiffness matrix and its applications for a timoshenko beam with mass and elastic axes eccentricity," Thin-Walled Structures, Article ID 107197, 2020.

[36] X. Liu, C. Sun, J. Ranjan Banerjee, H.-C. Dan, and L. Chang, "An exact dynamic stiffness method for multibody systems consisting of beams and rigid-bodies," Mechanical Systems and Signal Processing, vol. 150, Article ID 107264, 2021.

[37] H. Li, X. Yin, and W. Wu, "Dynamic stiffness formulation for in-plane and bending vibrations of plates with two opposite edges simply supported," Journal of Vibration and Control, vol. 24, no. 9, pp. 1652-1669, 2016.

[38] X. Liu and J. R. Banerjee, "Free vibration analysis for plates with arbitrary boundary conditions using a novel spectraldynamic stiffness method," Computers \& Structures, vol. 164, pp. 108-126, 2016.

[39] M. Nefovska-Danilovic and M. Petronijevic, "In-plane free vibration and response analysis of isotropic rectangular plates using the dynamic stiffness method," Computers \& Structures, vol. 152, pp. 82-95, 2015. 